\title{
Static Properties and Current-Driven Dynamics of Domain Walls in Perpendicular Magnetocrystalline Anisotropy Nanostrips with Rectangular Cross-Section
}

\author{
Eduardo Martinez \\ Universidad de Salamanca, Plaza de los Caidos s/n, 38008 Salamanca, Spain \\ Correspondence should be addressed to Eduardo Martinez, edumartinez@usal.es
}

Received 26 March 2012; Accepted 28 May 2012

Academic Editor: Giancarlo Consolo

Copyright () 2012 Eduardo Martinez. This is an open access article distributed under the Creative Commons Attribution License, which permits unrestricted use, distribution, and reproduction in any medium, provided the original work is properly cited.

\begin{abstract}
The current-induced domain wall motion along thin ferromagnetic strips with high perpendicular magnetocrystalline anisotropy is studied by means of full micromagnetic simulations and the extended one-dimensional model, taking into account thermal effects and edge roughness. A slow creep regime, where the motion is controlled by wall pinning and thermal activation, and a flow regime with linear variation of the DW velocity, are observed. In asymmetric stacks, where the Rashba spin-orbit field stabilizes the domain wall against turbulent transformations, the steady linear regime is extended to higher currents, leading to higher velocities than in single-layer or symmetric stacks. The pinning and depinning at and from a local constriction were also studied. The results indicate that engineering pinning sites in these strips provide an efficient pathway to achieve both high stability against thermal fluctuations and low-current depinning avoiding Joule heating. Finally, the current-driven dynamics of a pinned domain wall is examined, and both the direct and the alternating contributions to the induced voltage signal induced are characterized. It was confirmed that the direct contribution to the voltage signal can be linearly enhanced with the number of pinned walls, an observation which could be useful to develop domain-wall-based nano-oscillators.
\end{abstract}

\section{Introduction}

A typical pattern of a ferromagnetic sample consist on a set of domains and domain walls (DWs). The domains are uniformly magnetized regions, and DWs constitute the boundary between them. DWs have been intensively researched in the past, both in bulk or continuous films [1]. However, as many other areas of physics, the study of DWs has been revitalized by the advent of nanotechnology, and at the present, modern nanolithograpy techniques allow the fabrication of suitable ultra-thin ferromagnetic strips where DWs can be easily nucleated. The development of advance microscopy methods has also permitted them to be imaged and their dynamics to be explored. The traditional way to promote the DW dynamics is done by applying magnetic fields. Recently, a more promising alternative to drive DWs has been proposed. It consists on flowing electrical currents though ferromagnetic strip by using the novel physics of spin-transfer torque. Electrical current passing through a ferromagnetic strip becomes spin polarized along the local magnetization direction. When the current crosses a DW, spin angular momentum is transferred from the current to the magnetization, thereby inducing a torque which leads to DW motion. This spin-transfer torque phenomenon, which was firstly predicted by Berger [2, 3], has adiabatic and nonadiabatic contributions. The first one, which is expected to be dominant in wide walls, acts as a hard-axis field perpendicular to the magnetization inside the DW and controls the initial DW velocity. The nonadiabatic torque, which is expected to be dominant in thin wall, mimics an easy axis magnetic field and it is the responsible of the terminal DW velocity [4-8]. Although several experimental [9-23] and theoretical [24-29] studies have provided advances in the understanding of current-driven DW dynamics, there are many aspects of the underlying physical mechanism, such as the origin (large gradient of the local magnetization [3], spin-flip scattering $[5,8]$, or linear momentum transfer [6]) and the strength of the nonadiabatic contribution, which remain still unclear [30]. 
Apart from its intrinsic fundamental interest, the efficient control of the current-induced DW dynamics along thin strips is nowadays a promising but a technological challenge. Logic [31-33] and storage [34-36] devices based on DW displacement along thin ferromagnetic strips have been proposed during the last decade, where the DW position can be manipulated by means of constrictions which act as local pinning sites for the wall. These DW-based devices require a high efficient current-driven DW propagation, with high velocity under low current. The high stability of a trapped DW at a pinning site against thermal fluctuations, along with a low current DW depinning are also mandatory for recording applications. On the other hand, there exist other potential applications exploiting the current-induced DW dynamics, such as nano-oscillators [37-41] or nanosensors and amplifiers [42].

Most of the experimental and theoretical studies have been focused on soft Permalloy strips, which present several drawbacks for applications. For instance, critical depinning density currents around $1 \mathrm{~A} / \mu \mathrm{m}^{2}$ are required to promote the DW depinning from thermally stable pining notches $[13,14$, 26], but these currents remain too high for applications due to unwanted Joule heating effects. On the other hand, DWs in typical soft strips usually adopt wide (50-100 nm) and complex out-of-plane vortex configurations with low mobility $[14,15]$. The classical one-dimensional model (1 DM) of DW propagation provides a approach to understand experiments on soft strips, but its validity is rather limited because it cannot capture the full complexity of the vortex configuration nor its translational deformation, and timeconsuming micromagnetic simulations $(\mu \mathrm{M})$ are needed to interpret experimental measurements in the framework of available theories.

Due to these limitations, the attention is recently shifting to materials with high perpendicular magnetocrystalline anisotropy (PMA) resulting in an out-of-plane easy axis [4372]. Thin strips made of high PMA ferromagnetic materials are characterized by narrow DWs and combine several key advantages over soft magnetic materials, such as high nonadiabatic effects leading to lower critical current densities and high DW velocities. A pinned DW at constrictions in these high PMA strips also depicts high stability against thermal fluctuations and low current-induced DW depinning against Joule heating. All these observations make high PMA strips very attractive, not only for technological applications, but also as valuable systems to test the microscopic theories of the spin-transfer torque by means of the simple one-dimensional models. This work reviews recent theoretical and numerical results in this class of high PMA materials, and it discusses the relevant implications they entail for the nature of the current-driven DW dynamics and its potential technological applications. The paper is organized as follows. The typology of the DWs in strips with high PMA of rectangular crosssection, along with a brief description of the micromagnetic and the one dimensional models are described in Section 2. The current-driven domain wall dynamics along both a freedefect strip and other with edge roughness is studied in Section 3. Section 4 is dedicated to the analysis of pinning of a DW in a geometrical constriction of the strip, and its depinning under both static fields and currents. The pinned DW oscillations driven by static currents and the possibility of developing DW-based oscillators are evaluated in Section 5. Finally, the main conclusions of the study, along with the theoretical open questions and future numerical tasks are summarized in Section 6.

\section{Geometry, Materials and Models}

We focus our attention on thin strips of rectangular crosssection $L_{y} \times L_{z}$ with the easy axis along the $z$-axis $\left(\vec{u}_{K}=\right.$ $\vec{u}_{z}$, see Figure $\left.1(\mathrm{a})\right)$. In order to mimic a material with high perpendicular anisotropy, the following parameters have been considered: saturation magnetization $M_{s}=3 \times$ $10^{5} \mathrm{~A} / \mathrm{m}$, exchange constant $A=10^{-11} \mathrm{~J} / \mathrm{m}$, and anisotropy constant $K=2 \times 10^{5} \mathrm{~J} / \mathrm{m}^{3}$. According to Weller et al. [73], these parameters correspond to a typical CoPtCr alloy. The dimensionless damping parameter was taken to be $\alpha=0.2$, which is in the same order of magnitude as the materials with high PMA analysed by Metaxas et al. [74]. A standard finite-difference scheme with cubic computational cells of $\Delta x=3 \mathrm{~nm}$ in side was considered.

Two equilibrium states can be found depending on the width $\left(L_{y}\right)$ and thickness $\left(L_{z}\right)$ of the strip: Bloch DW and Neel DW, which are plotted in Figures 1(b) and 1(c), respectively. In both cases, the magnetization inside the DW rests in the $x y$-plane, and it points along the $y$-axis and $x$-axis for Bloch $\left(\vec{m}_{\text {DW }} \propto \pm \vec{u}_{y}\right)$ and Neel $\left(\vec{m}_{\text {DW }} \propto \pm \vec{u}_{x}\right)$ configurations, respectively. These magnetic configurations were the initial state of a minimization energy process in order to evaluate which is the equilibrium state with minimum energy for different values of $L_{y}$ and $L_{z}$. Figure $1(\mathrm{~d})$ shows the critical value of $L_{y}$ as a function of $L_{z}$ above which the Bloch configuration has smaller energy than the Neel one. The transition between Bloch and Neel configurations moves toward smaller values of $L_{y}$ as the strip thickness $L_{z}$ increases.

In order to analyse the DW dynamics from a full-micromagnetic model $(\mu M)$ point of view, the strip is assumed to be infinite along the $x$-axis, and a moving computational region centered on the DW with $L_{x}=1.2 \mu \mathrm{m}$ in length was performed [26]. Starting from the corresponding equilibrium state at rest, either Bloch or Neel, the response to the action of magnetic fields along the easy axis $\left(\vec{B}_{e}=B_{e} \vec{u}_{z}\right)$ and/or electrical density currents along the $x$-axis $\vec{j}_{a}=$ $j_{a} \vec{u}_{x}$, both of them spatially uniform and instantaneously applied at $t=0$, is micromagnetically $(\mu M)$ evaluated by numerically solving the Langevin-Landau-Lifshitz-Gilbert equation augmented by the adiabatic and nonadiabatic spinpolarized torques $[5,8,26]$ :

$$
\begin{aligned}
\frac{d \vec{m}}{d t}= & -\gamma_{0} \vec{m} \times\left(\vec{H}_{\mathrm{eff}}+\vec{H}_{\mathrm{th}}\right)+\alpha\left(\vec{m} \times \frac{d \vec{m}}{d t}\right) \\
& +\frac{\mu_{B} P}{e M_{s}}\left[\left(\overrightarrow{j_{a}} \cdot \nabla\right) \vec{m}-\xi \vec{m} \times\left(\overrightarrow{j_{a}} \cdot \nabla\right) \vec{m}\right],
\end{aligned}
$$

where $\vec{m}(\vec{r}, t)=\vec{M} / M_{s}$ is the normalized local magnetization, $\gamma_{0}$ is the gyromagnetic ratio, and $\vec{H}_{\text {eff }}$ is the effective field, which includes exchange, self-magnetostatic, uniaxial 


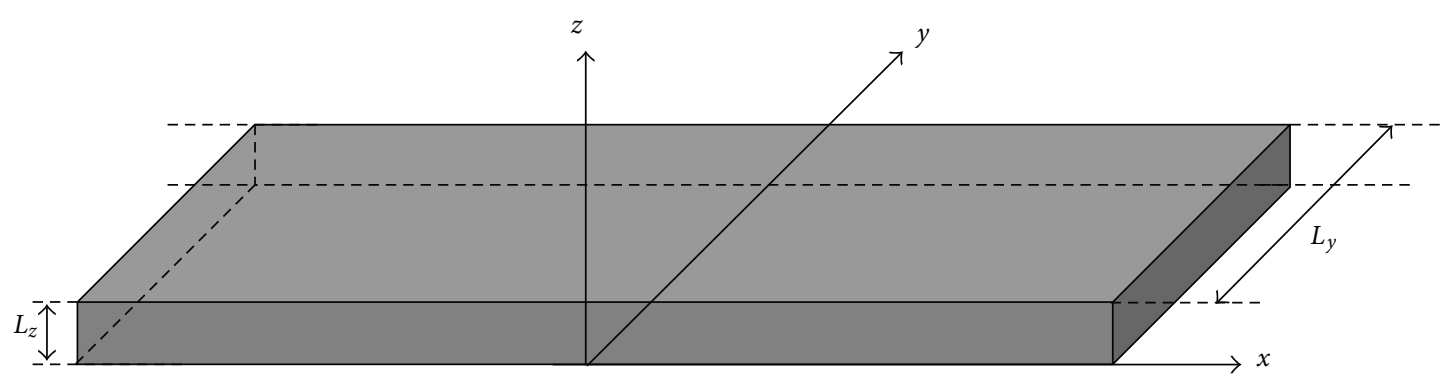

(a)

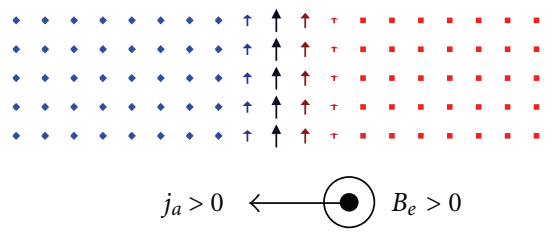

(b) Bloch DW

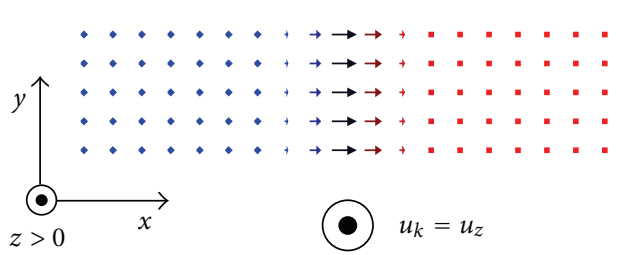

(c) Neel DW

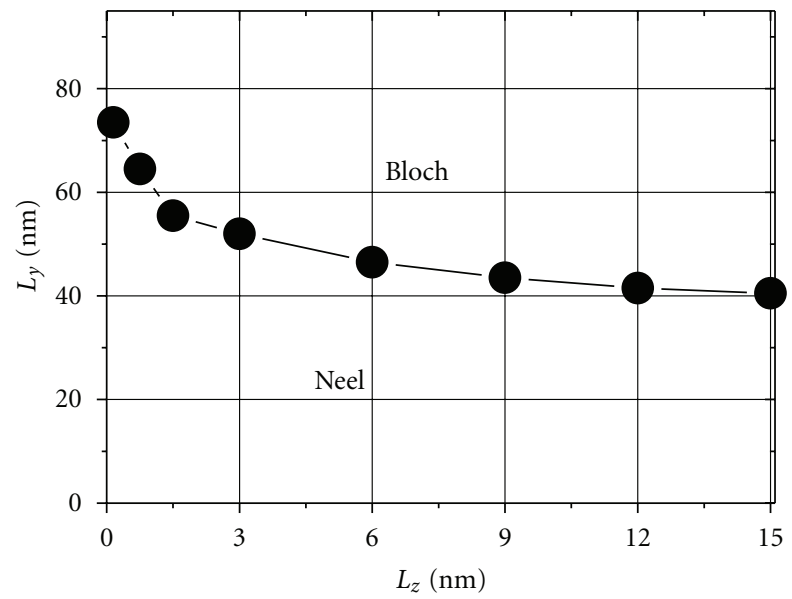

(d)

Figure 1: (a) Scheme of the rectangular cross-section strip geometry. Equilibrium states for a DW in thin CoPtCr strips: (b) Bloch, (c) Neel. (d) Phase diagram of the equilibrium state as a function of the width $L_{y}$ and the thickness $L_{z}$ of the strip (from [41]).

anisotropy ( $\vec{u}_{K}=\vec{u}_{z}$ is easy axis), and external field contributions [26]. $\vec{H}_{\text {th }}$ is the thermal field [26], which is a Gaussian random process with the following statistical properties $[75,76]$ :

$$
\begin{gathered}
\left\langle H_{\mathrm{th}, i}(\vec{r}, t)\right\rangle=0, \\
\left\langle H_{\mathrm{th}, i}(\vec{r}, t) H_{\mathrm{th}, j}\left(\vec{r}^{\prime}, t^{\prime}\right)\right\rangle=2 D_{\mu M} \delta_{i j} \delta\left(\vec{r}-\vec{r}^{\prime}\right) \delta\left(t-t^{\prime}\right) .
\end{gathered}
$$

Equation (2) indicates that the average of the thermal field taken over different stochastic realizations vanishes in each direction $i: x, y, z$. The thermal field $\vec{H}_{\text {th }}$ is assumed to be uncorrelated in time $\left(\delta\left(t-t^{\prime}\right)\right)$ and uncorrelated at different points $\delta\left(\vec{r}-\overrightarrow{r^{\prime}}\right)$ of the finite difference mesh, as stated by (3). The strength of the thermal field, which follows from the fluctuation-dissipation theorem $[75,76]$, is given by

$$
D_{\mu M}=\frac{\alpha K_{B} T}{\gamma_{0} \mu_{0} M_{s}},
$$

where $K_{B}$ is the Boltzmann constant, and $T$ represents the temperature.

The last two terms on the right side of (1) represent the adiabatic and the nonadiabatic spin-transfer torques, respectively [5], where $\mu_{B}$ is the Bohr magneton, $e<0$ the electron's electric charge, and $P$ is the spin polarization factor of the current, which here is assumed to be $P=0.5$. The coefficient $\xi$ is a dimensionless constant describing the degree of nonadiabaticity between the spin of conduction electrons and the local magnetization [5]. Equation (1) is numerically solved by means of a fourth-order Runge-Kutta scheme. The micromagnetic results described hereafter were obtained by using a time step of $0.15 \mathrm{ps}$, and it was verified in several tested cases that a time step of 0.1 ps does not modify the presented results. Although the following micromagnetic results were computed with a cell size of $\Delta x=3 \mathrm{~nm}$, it was also checked that the results do not significantly change when the cell size is reduced to half for several tested cases.

On the other hand, the DW dynamics has been also analyzed from the one-dimensional model (1 DM) point of view, which is described by the following equations $[15,29$, 63]:

$$
\begin{aligned}
\left(1+\alpha^{2}\right) \frac{\dot{X}}{\Delta}= & \alpha \gamma_{0}\left(H_{e}+H_{p}(X)+H_{\mathrm{th}}(t)\right) \\
& +\frac{1}{2} \gamma_{0} H_{K} \sin (2 \Phi)-(1+\alpha \xi) \frac{1}{\Delta} \frac{\mu_{B} P}{e M_{s}} j_{a} \\
\left(1+\alpha^{2}\right) \dot{\Phi}= & \gamma_{0}\left(H_{e}+H_{p}(X)+H_{\mathrm{th}}(t)\right) \\
& -\alpha \frac{1}{2} \gamma_{0} H_{K} \sin (2 \Phi)-(\xi-\alpha) \frac{1}{\Delta} \frac{\mu_{B} P}{e M_{s}} j_{a}
\end{aligned}
$$




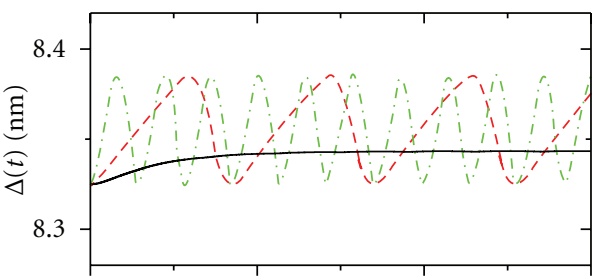

(a)

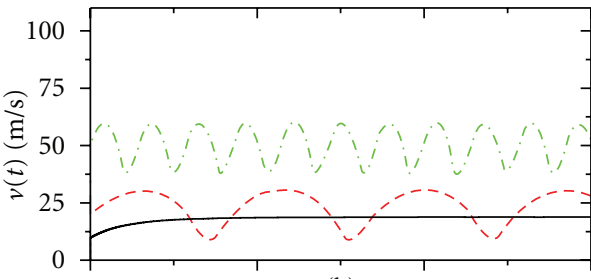

(b)

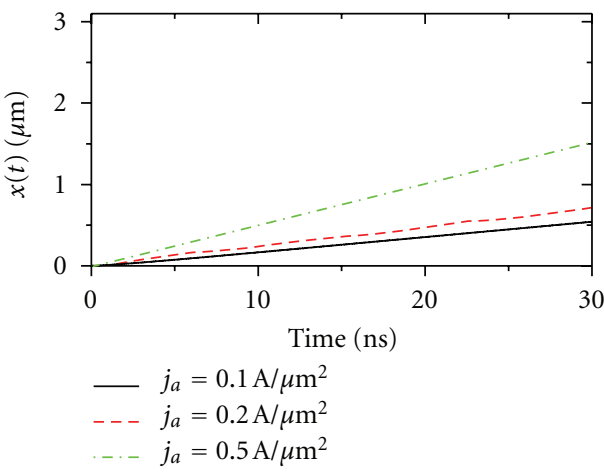

(c)

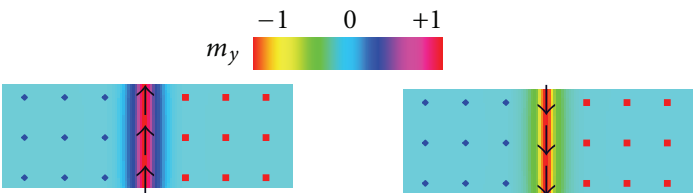

(d)

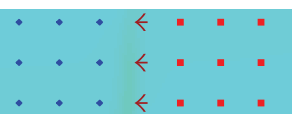

(e)

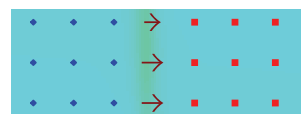

(g)

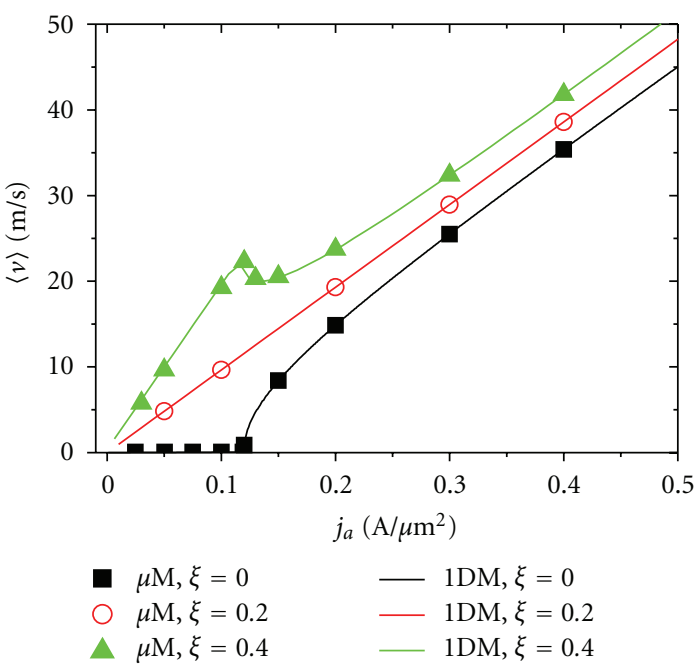

(h)

Figure 2: Current driven DW dynamics along a perfect strip with $L_{y} \times L_{z}=120 \times 3 \mathrm{~nm}^{2}$. (a), (b), and (c) depict the micromagnetically $(\mu M)$ computed temporal evolution of the DW width $\Delta$, the DW velocity $v$, and the DW position $x$ under three different density currents: $j_{a}=0.1 \mathrm{~A} / \mu \mathrm{m}^{2}, j_{a}=0.2 \mathrm{~A} / \mu \mathrm{m}^{2}$, and $j_{a}=0.5 \mathrm{~A} / \mu \mathrm{m}^{2}$ for a nonadiabatic parameter of $\xi=0.4$. (d) $-(\mathrm{g})$ show typical micromagnetic states of the propagating DW for currents larger than the Walker breakdown $\left(j_{a}>j_{W}(\xi)\right)$. (h) Time-averaged DW velocity as function of $j_{a}$ for three different values of the nonadiabatic parameter: $\xi=0, \xi=0.2$ and $\xi=0.4$. Dots correspond to $\mu M$ results, whereas lines are predictions from the $1 \mathrm{DM}$ with $\Delta=8.325 \mathrm{~nm}$ and $H_{K}=12533.5 \mathrm{~A} / \mathrm{m}$. (Reprinted with permission From [70, 72]. Copyright (2012), American Institute of Physics).

where $X=X(t)$ is the position of the DW centre, $\Phi=\Phi(t)$ is the tilt angle of the DW magnetization, $\Delta$ is the DW width, and $H_{K}$ is the hard-axis anisotropy field of magnetostatic origin. $H_{e}=B_{e} / \mu_{0}$ is the applied field along the easy $z$-axis, and $H_{p}(X)$ is the spatial dependent pinning field, which can be expressed as $H_{p}(X)=-\left(1 / 2 \mu_{0} M_{s} L_{y} L_{z}\right)\left(\partial V_{\text {pin }}(X) / \partial X\right)$, where $V_{\text {pin }}(X)$ is the local pinning potential. $H_{\text {th }}(t)$ is a stochastic random thermal field which describes the effect of thermal fluctuation in the $1 \mathrm{DM}[24,26,27,29]$. The thermal field is assumed to be a Gaussian-distributed stochastic process with zero mean value $\left(\left\langle H_{\mathrm{th}}(t)\right\rangle=0\right)$ and uncorrelated in time $\left(\left\langle H_{\text {th }}(t) H_{\text {th }}\left(t^{\prime}\right)\right\rangle=2 D_{1 D} \delta\left(t-t^{\prime}\right)\right)$. The factor $D_{1 D}$ represents the strength of the thermal field, which can be obtained from the fluctuation-dissipation theorem $\left(D=\left(\alpha K_{B} T / \mu_{0} V_{\mathrm{DW}} M_{s} \gamma_{0}\right)^{1 / 2}\right.$, where $V_{\mathrm{DW}}=\Delta L_{y} L_{z}$ is the DW volume).

\section{Current-Driven DW Dynamics}

The essential goal for designing recording and logic DWbased devices which can be competitive with nowadays available technologies is to efficiently improve the velocity of the propagating DW with low currents against unwanted Joule heating effects. This section is dedicated to describe the DW dynamics along high PMA strips driven by spinpolarized current, from the ideal or perfect strips to the realistic ones, where disorder and thermal effects play a significant role.

3.1. Perfect Strips at Zero Temperature. The case of a perfect strip with $L_{y} \times L_{z}=120 \times 3 \mathrm{~nm}^{2}$ is firstly considered. Figures 2(a), 2(b), and 2(c) depicts the micromagnetically $(\mu M)$ computed temporal evolution of the DW width $\Delta$, the DW velocity $v$ and the DW position $x$ under three different density currents: $j_{a}=0.1 \mathrm{~A} / \mu \mathrm{m}^{2}, j_{a}=0.2 \mathrm{~A} / \mu \mathrm{m}^{2}$ and $j_{a}=0.5 \mathrm{~A} / \mu \mathrm{m}^{2}$ for a nonadiabatic parameter of $\xi=$ 0.4 . The instantaneous DW width was numerically evaluated considering the Thiele's definition [77], which is given by $1 / \Delta(t)=\left(1 / 2 L_{y} L_{z}\right) \int(\partial \vec{m}(\vec{r}, t) / \partial x)^{2} d V$. For $j_{a}=0.1 \mathrm{~A} / \mu \mathrm{m}^{2}$, which is below than the Walker breakdown $\left(j_{W}(\xi=0.4)=\right.$ $0.12 \mathrm{~A} / \mu \mathrm{m}^{2}$ ), the DW moves by preserving its initial Bloch 
configuration and reaching a stationary linear behavior with a steady DW velocity. In contrast, for $j_{a}=0.2 \mathrm{~A} / \mu \mathrm{m}^{2}>$ $j_{W}(0.4)$, the DW does not reach a steady regime and its internal magnetization precesses periodically around the easy $z$-axis. Figures 2(d)-2(g) depict typical Bloch and Neel configurations adopted by the DW during the turbulent precessional regime.

The time-averaged DW velocity $\langle v\rangle$ as a function of $j_{a}$ is shown in Figure 2(h) for three different values of the nonadiabatic parameter: $\xi=0$ (perfect adiabatic case), $\xi=0.2$, and $\xi=0.4$. Dots correspond to $\mu M$ results and lines to $1 \mathrm{DM}$ predictions, where the value of DW width $\Delta=8.325 \mathrm{~nm}$ was obtained from the micromagnetic configuration of the DW at rest, and the hard axis anisotropy field of magnetostatic origin $H_{K}=12533.5 \mathrm{~A} / \mathrm{m}$ was deduced from the micromagnetically computed Walker field $B_{W}=$ $\mu_{0} H_{W} \approx 1.575 \mathrm{mT}\left(H_{K}=2 H_{W} / \alpha\right)$. In the perfect adiabatic case $(\xi=0)$, there is a threshold density current $\left(j_{W}(\xi=\right.$ $0)$ ) below which the DW motion is not achieved. Above $j_{W}(0)$, the motion takes place by means of DW precession similarly to the field-driven case above the Walker breakdown $[66,70]$. In the $1 \mathrm{DM}$, the Walker field $H_{W}$ and the Walker threshold current $\left(j_{W}\right)$ are related by $j_{W}(\xi)=(1 /|\xi-\alpha|)$ $\left(|e| M_{s} / P \mu_{B}\right) \gamma_{0} \Delta H_{W}$, which yields $j_{W}(0)=0.12 \mathrm{~A} / \mu \mathrm{m}^{2}$, in good agreement with full micromagnetic results $(\mu M)$. In the nonadiabatic case $(\xi>0)$, the DW moves for any positive current along the perfect strip, and it does it without changing its initial structure if the nonadiabatic parameter matches the damping parameter $(\xi=\alpha=0.2)$. Note that $j_{W}(\xi=\alpha)=\infty$. For any other case $(0<\xi \neq \alpha)$, there is a Walker threshold density current $j_{W}(\xi)$ above which the DW rotates around the $z$-axis similarly to the field-driven case. The current DW mobility is defined as $\mu=d v / d j_{a}$, and it is given by $\mu_{\mathrm{st}, j}=(\xi / \alpha)\left(\mu_{B} P /|e| M_{s}\right)$ and $\mu_{\mathrm{hf}, j}=((1+$ $\left.\xi \alpha) /\left(1+\alpha^{2}\right)\right)\left(\mu_{B} P /|e| M_{s}\right)$ for the steady $\left(j_{a}<j_{W}\right)$ and the high-current $\left(j_{a} \gg j_{W}\right)$ linear regimes [25], respectively.

\subsection{Rough Strips at Room Temperature. Former simulations} were conducted on a perfect strip, without any defect which could prevent the free DW motion. However, due to the nanolithography fabrication process, realistic strips have defects and imperfections such as edge roughness [78] which oppose to the free DW motion. Due to the electron beam, the edge roughness in real samples can be characterized by an average depth (along the $y$-axis) and an average length (along the $x$-axis). In order to mimic the irregularities in the sample geometry originating from electron beam lithography, natural edge roughness is modelled by independently deforming the perfect finite difference mesh at both edges of the strip [26]. Here, a random roughness pattern, where both the depth and the length are assumed to be equal to the typical grain size $D_{g}=3 \mathrm{~nm}$, is considered, so each computational cell at the edges of the strip is or not magnetic with $50 \%$ of probability. A $6 \mu \mathrm{m}$-long strip pattern is generated at the beginning of the simulation to be used by the moving $1.2 \mu \mathrm{m}$ long computational region.

Typical examples of the micromagnetic results for the temporal evolution of the DW position along a rough strip are depicted in Figure 3 for three different currents: (a) $j_{a}=$
$0.04 \mathrm{~A} / \mu \mathrm{m}^{2}$, (b) $j_{a}=0.1 \mathrm{~A} / \mu \mathrm{m}^{2}$, and (c) $j_{a}=0.2 \mathrm{~A} / \mu \mathrm{m}^{2}$, considering a nonadiabatic parameter of $\xi=0.4$ and temporal window of $t_{w}=50 \mathrm{~ns}$. The deterministic trajectory ( $T=0$, dashed lines) and ten different stochastic realizations at room temperature $(T=300 \mathrm{~K}$, solid lines $)$ are shown for each current Computing ten stochastic realizations each one of 50ns requires a enormous computational effort in the framework of the full micromagnetic model $(\mu \mathrm{M})$, and in every specific case we must wonder if this number is sufficient to obtain statistically meaning results. In order to justify this choice, the same problem was also analyzed in the 1DM (see Figure 3(h)), where the number of stochastic realizations and the temporal window can be increased by one or two orders of magnitude with reduced computational effort. It was confirmed that results of extended $1 \mathrm{DM}$ simulations with $t_{w}=500 \mathrm{~ns}$ and $N=100$ are quite similar to the ones obtained for $t_{w}=50 \mathrm{~ns}$ and $N=10$, and they are also in quantitative agreement with the full micromagnetic results. This observation allows us to justify that $N=10$ is sufficient to obtain meaningful statistically results. The deterministic threshold current $j_{d}$, defined as the minimum current required to promote the sustained DW propagation at zero temperature, is around $0.1 \mathrm{~A} / \mu \mathrm{m}^{2}$ for a $L_{y} \times L_{z}=$ $120 \times 3 \mathrm{~nm}^{2}$ strip with a typical roughness size of $D_{g}=3 \mathrm{~nm}$.

For very low currents, the DW does not depart from its initial position (see Figure 3(a)) because the force on the DW due to the applied current $\left(j_{a}=0.04 \mathrm{~A} / \mu \mathrm{m}^{2}\right)$ is still too low to overcome the energy barrier induced by the roughness, even at room temperature. For larger currents but smaller than the deterministic depinning threshold $\left(0.05 \mathrm{~A} / \mu \mathrm{m}^{2} \leq j_{a} \leq j_{d}(\xi)\right)$, the DW also gets pinned due to the roughness, but due to thermal fluctuations, it eventually depins and propagates along the strip (see Figure $3(\mathrm{~b})$ ). In this thermally activated field regime $\left(0.05 \mathrm{~A} / \mu \mathrm{m}^{2} \leq\right.$ $j_{a} \leq j_{d}(\xi)$ ), the DW displaces several nanometers from its initial position for some time before reaching a region of higher surface roughness where it becomes pinned again for some time up to thermal fluctuations assist again the DW depinning and propagation. Similar to the field-driven case, the DW follows a creep regime, where it can be seen as a thermally activated interface that is creeping over local pinning sites. For very high currents $\left(j_{a} \geq 0.2 \mathrm{~A} / \mu \mathrm{m}^{2}\right)$, the DW position increases almost linearly as the time elapses for all the stochastic realizations, and it reaches a quite similar final position at the end of the evaluated temporal window $\left(t_{w}=50 \mathrm{~ns}\right.$, see Figure 3(c)). Therefore, in such a high regime, the DW dynamics is governed by the current, which is high enough to overcome the energy barrier of the roughness independently on the thermal effects. It is worthy to note that, independently of the nonadiabatic parameter, the thermally activated DW motion along the rough strip takes place by precessing between Bloch and Neel configurations. These characteristic configurations are shown in Figures 3(d)-3(g). This observation is in contrast to the DW propagation along a perfect strip, where DW propagates rigidly for any finite current if the nonadiabatic parameter is equal to the damping $(\xi=\alpha)$.

The statistically averaged DW velocity $[\langle v\rangle]$ as a function of $j_{a}$ along a rough strip at room temperature $(T=300 \mathrm{~K})$ is 


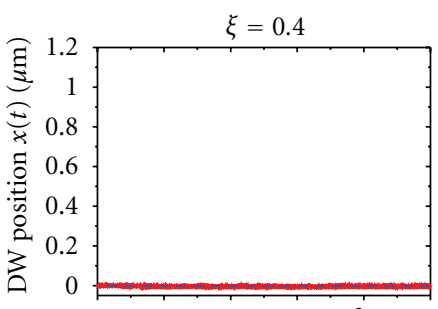

(a) $j_{a}=0.04 \mathrm{~A} / \mu \mathrm{m}^{2}$

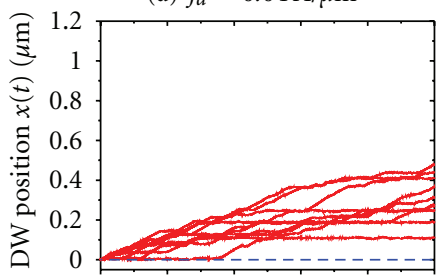

(b) $j_{a}=0.1 \mathrm{~A} / \mu \mathrm{m}^{2}$

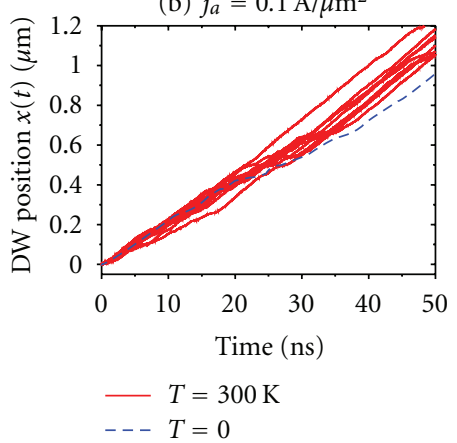

(c) $j_{a}=0.2 \mathrm{~A} / \mu \mathrm{m}^{2}$

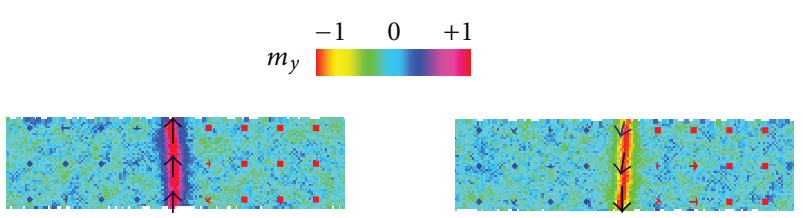

(d)

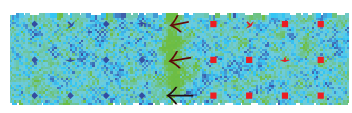

(e)

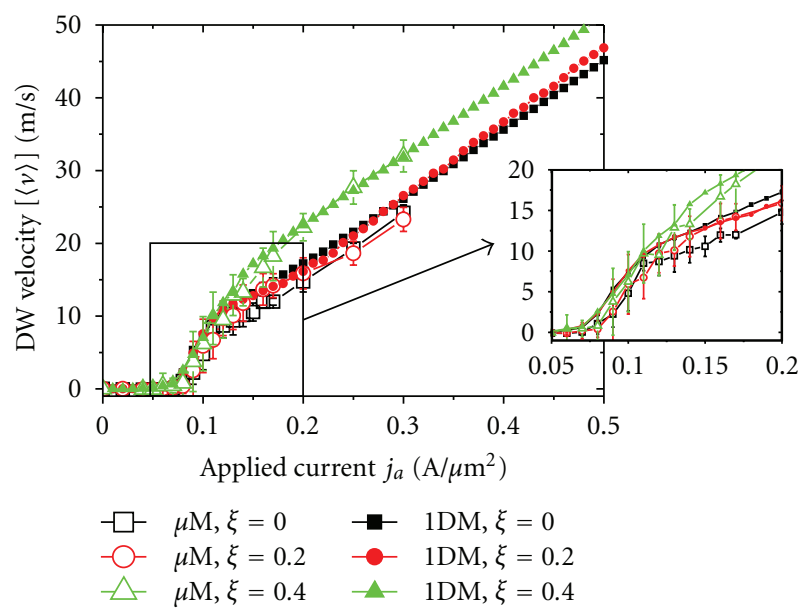

(h)

FIgURE 3: Current driven DW dynamics along a rough $\left(D_{g}=3 \mathrm{~nm}\right)$ strip with $L_{y} \times L_{z}=120 \times 3 \mathrm{~nm}^{2}$. Micromagnetically ( $\mu M$ ) computed temporal evolution of the DW position $x$ under three different density currents: (a) $j_{a}=0.04 \mathrm{~A} / \mu \mathrm{m}^{2},(\mathrm{~b}) j_{a}=0.1 \mathrm{~A} / \mu \mathrm{m}^{2}$ and $(\mathrm{c}) j_{a}=$ $0.2 \mathrm{~A} / \mu \mathrm{m}^{2}$ for a nonadiabatic parameter of $\xi=0.4$. Blue-dashed lines correspond to the deterministic case $(T=0)$, whereas red-solid lines correspond to ten stochastic realizations at room temperature $(T=300 \mathrm{~K})$. (d) $-(\mathrm{g})$ show typical micromagnetic states of the propagating DW along a rough strip at room temperature. (h) Time-averaged DW velocity as function of $j_{a}$ for three different values of the nonadiabatic parameter: $\xi=0, \xi=0.2$, and $\xi=0.4$. Open-dots correspond to $\mu M$ results (with indication of the standard deviation), whereas solid symbols are the predictions from the $1 \mathrm{DM}$ with $\Delta=8.325 \mathrm{~nm}, H_{K}=12533.5 \mathrm{~A} / \mathrm{m}$, and a periodic pinning potential given by $V_{\text {pin }}(X)=$ $V_{0} \sin ^{2}(\pi X / p)$ with $V_{0}=1.65 \times 10^{-20} \mathrm{~J}$ and $p=30 \mathrm{~nm}$. The inset shows the results around between $j_{a}=0.05 \mathrm{~A} / \mu \mathrm{m}^{2}$ and $j_{a}=0.2 \mathrm{~A} / \mu \mathrm{m}^{2}$ (Reprinted with permission From [70]. Copyright (2012), American Institute of Physics).

shown in Figure 3(h) for three different values of the nonadiabatic parameter: $\xi=0, \xi=0.2$, and $\xi=0.4$. This statistically averaged DW velocity $[\langle v\rangle]$ was calculated by firstly averaging over a temporal window of $t_{w}=50 \mathrm{~ns}$ as $\langle v\rangle_{i}=\left(1 / t_{w}\right) \int_{0}^{t_{w}} v(t) d t$, and then, averaging over ten $(N=$ 10) stochastic realizations as $[\langle v\rangle]=(1 / N) \sum_{i=1}^{N}\langle v\rangle_{i}$. Open symbols correspond to $\mu M$ simulations of a rough strip with $D_{g}=3 \mathrm{~nm}$, and full symbols were obtained in the framework of the 1DM, where the local pinning landscape is modeled by a periodic pinning potential given by $V_{\text {pin }}(X)=$ $V_{0} \sin ^{2}(\pi X / p)$. The values of the energy barrier $V_{0}$ and the spatial periodicity $p$ were chosen to match a deterministic depinning field similar to the micromagnetic value for $D_{g}=$ $3 \mathrm{~nm}: V_{0}=1.65 \times 10^{-20} \mathrm{~J}$, and $p=30 \mathrm{~nm}$. Figure $3(\mathrm{~h})$ confirms that the velocity-current characteristic predicted by the $1 \mathrm{DM}$ is in good qualitative and quantitative agreement with full $\mu M$ results.

Analogously to the creep and flow regimes seen in former numerical studies and other experimental studies of field-driven motion, the DW mobility increases at low currents and saturates at higher values (see Figure 3(h)). Therefore, the inclusion of surface roughness along with thermal fluctuations in both $\mu M$ and $1 \mathrm{DM}$ simulations provides a proper explanation to understand experimental observations. Apart from the qualitative agreement with experiments $[47,55]$, micromagnetic simulations also provide information on the internal DW structure during its motion. In the single layer rough strip, the thermally activated DW translation occurs by DW precession around the $z$-axis, even for the case of $\xi=\alpha=0.2$. In the creep regime $\left(0.05 \mathrm{~A} / \mu \mathrm{m}^{2}<j_{a}<0.1 \mathrm{~A} / \mu \mathrm{m}^{2}\right),[\langle v\rangle]$ increases exponentially with $j_{a}$, but it does not depend significantly on nonadiabatic parameter (see Figure $3(\mathrm{~h})$ ), so it is dominated by roughness and thermal fluctuations, and $\xi$ does not play a remarkable role. In the high-current flow regime $\left(j_{a} \gg 0.1 \mathrm{~A} / \mu \mathrm{m}^{2}\right)$, the DW mobility recovers the deterministic value for the perfect strip $\left(\mu_{h f, j}=((1+\xi \alpha) /(1+\right.$ $\left.\left.\left.\alpha^{2}\right)\right)\left(\mu_{B} P /|e| M_{s}\right)\right)$, both for $\xi=0$ and $\xi=0.4$ cases. However, 
as it is clear in Figure 3(h), the DW mobility in the highcurrent flow regime for $\xi=\alpha=0.2$ approaches to the one of the perfect adiabatic case $\left(\mu_{h f, j}=\left(1 /\left(1+\alpha^{2}\right)\right)\left(\mu_{B} P /|e| M_{s}\right)\right)$. These numerical predictions point out that, in a single layer strip, the surface roughness favors the turbulent motion with DW precession between Bloch and Neel configurations. It is also clear in Figure 3(h) that the standard deviation of $[\langle v\rangle]$ versus $j_{a}$ is enlarged in the creep regime, whereas it decreases significantly as $j_{a}$ increases in the high-current flow regime. This fact indicates that at high currents, the DW dynamics becomes insensitive to the roughness, being mainly dominated by the current force.

\subsection{Strips Sandwiched in Asymmetric Stacks: The Role of the} Spin-Orbit Interaction. Experimental measurements along high PMA strips have been performed in several architectures, ranging from single layer $[50,53]$ to multilayer stacks $[47,49,51,52,54,55]$. Former section was dedicated to the study of the current-driven DW dynamics along a single layer PMA strip. However, the experiments on DW propagation along a high PMA Cobalt strip sandwiched between two dissimilar nonmagnetic layers $(\mathrm{Pt} / \mathrm{Co} / \mathrm{AlO})$ are particularly interesting because they have pointed out a high spin-torque efficiency leading to very high DW velocities at reduced currents $[51,55]$. The suggested reason for this behavior is the spin-orbit interaction (SOI) on the conduction electrons, which originates from the structural inversion asymmetry (SIA) of the multilayer stack. The SOI allows for the transfer of orbital angular momentum from the crystal lattice to the local magnetization [79], and it is mediated by an effective Rashba magnetic field $\left(\vec{H}_{R}\right)$ which is given by $[55,80,81]$

$$
\vec{H}_{R}=\frac{\alpha_{R} P}{\mu_{0} \mu_{B} M_{s}}\left(\vec{u}_{z} \times \vec{j}_{a}\right)
$$

where $\vec{u}_{z}$ is the unit vector along the perpendicular axis (the $z$ direction) and $\alpha_{R}$ is the Rashba parameter which describes the strength of the SOI [81]. The aim of the present section consists on analysing the current-driven DW propagation in the presence of the Rashba field. This field is added as a new contribution to the effective field in (1) considering a Rashba parameter of $\alpha_{R}=10^{-11} \mathrm{eVm}$, which is a typical value of a two-dimensional electron gas with SIA [55, 80, 81]. The $\mu M$ results along a perfect strip are collected in Figure 4, which depicts the temporal evolution of the DW width [77] $\Delta(t)$, the DW velocity $v(t)$, and the DW position $x(t)$, respectively, under three different values of $j_{a}$ for the case of finite Rashba field $\left(\alpha_{R}=10^{-11} \mathrm{eVm}\right)$ with $\xi=0.4$. Contrary to what happens in the absence of Rashba field for currents larger than the Walker breakdown (see Figures 2(a)-2(c)), the DW reaches a stationary behavior for all the studied currents $\left[0,1 \mathrm{~A} / \mu \mathrm{m}^{2}\right]$, and it propagates rigidly with steady velocity. Typical steady Bloch DW configurations reached under two values of $j_{a}$ are depicted in Figures 4(d) and 4(e).

The time-averaged DW velocity $\langle v\rangle$ over a temporal window of $t_{w}=50 \mathrm{~ns}$ is depicted as a function of $j_{a}$ in Figure 4(f) for several values of $\xi$ in the case of a perfect strip $\left(D_{g}=0\right)$ at zero temperature $(T=0)$. Open symbols correspond to the case of finite Rashba field with $\alpha_{R}=$ $10^{-11} \mathrm{eVm}$. The results corresponding to zero-Rashba field $\left(\alpha_{R}=0\right.$, filled symbols $)$ of former Figure $2(\mathrm{~h})$ are also included for comparison. In the perfect adiabatic case $(\xi=$ 0 ), a minimum density current of $j_{W}(\xi=0) \approx 0.12 \mathrm{~A} / \mu \mathrm{m}^{2}$ is required to promote self-sustained DW motion in absence of Rashba field $\left(\alpha_{R}=0\right)$. Above this intrinsic critical current, the DW moves turbulently by precessing clockwise around the $z$-axis between Bloch and Néel configurations, and for very high currents $\left(j_{a} \gg j_{W}(\xi=0)\right)$ the DW mobility, which is defined as $\mu=d v / d j_{a}$, is $\mu_{t}(\xi=0)=(1 /(1+$ $\left.\left.\alpha^{2}\right)\right)\left(\mu_{B} P /|e| M_{s}\right)[25]$, which tends to $\mu_{t}(\xi=0)=\mu_{B} P /|e| M_{s}$ for $\alpha \ll 1$. However, when a finite Rashba field with $\alpha_{R}=$ $10^{-11} \mathrm{eVm}$ is taken into account, no DW motion is achieved in the perfect adiabatic case $(\xi=0)$ even for high currents such as $j_{a}=1 \mathrm{~A} / \mu \mathrm{m}^{2}$. Therefore, $\vec{H}_{R}$ increases the critical intrinsic current in the perfect adiabatic case $(\xi=0)$. For $\xi=$ $\alpha=0.2$, the DW velocity increases linearly with $j_{a}$ for any finite value, and the DW mobility is $\mu_{s}(\xi=\alpha)=\mu_{B} P /|e| M_{s}$ independently of the Rashba field. Note that this mobility is similar to the one achieved in the high current turbulent regime $\left(j_{a} \gg j_{W}(\xi)\right)$ for zero-Rashba field independently on $\xi$. Finally, for $\xi=0.4$, the DW precesses turbulently and counter-clockwise between Bloch and Néel configurations above the Walker threshold at zero Rashba field $\left(\alpha_{R}=0\right)$, and under currents well above than this threshold the DW mobility approaches again to one observed for both $\xi=0$ and $\xi=0.2$ cases $\left(\mu_{t}(\xi) \approx \mu_{B} P /|e| M_{s}\right.$, for $\left.\alpha \cong \xi \ll 1\right)$. However, there is no Walker breakdown in the presence of the Rashba field $\left(\alpha_{R}=10^{-11} \mathrm{eVm}\right)$ for $\xi \neq 0$, and the DW mobility maintains the value of the linear low-current steady regime $\left(\mu_{s}(\xi>0)=(\xi / \alpha)\left(\mu_{B} P /|e| M_{s}\right)\right)$ in the whole analyzed range of density currents.

The effect of the Rashba field under positive current $\left(j_{a}>\right.$ 0 ) is equivalent to a homogeneous transverse field along $y>0$-axis, and both of them promote the stabilization of the Bloch up DW configuration by raising the energy barrier against transformations to Néel DW. If the current is reversed $\left(j_{a}<0\right)$, the Rashba field points along the negative transverse direction $(y<0)$, and similarly to a negative transverse magnetic field, it firstly promotes the transition from Bloch up to Bloch down. Once this transition is completed, the Bloch down DW moves rigidly in the opposite sense $(x<0)$ [55]. These results indicate that due to the SOI in a asymmetric trilayer stack with SIA $\left(\vec{H}_{R} \neq 0\right)$, the linear steady high mobility regime is extended to high currents, and therefore, it allows to achieve rigid DW propagation with higher DW velocities than the ones achieved in a single layer or a symmetric multilayer stack, where the Rashba field due to the SOI is negligible $\left(\vec{H}_{R}=0\right)$ and the maximum velocity is limited by the nonadiabatic parameter.

In order to get a more realistic description, and as it was done for a single layer strip $\left(\vec{H}_{R}=0\right)$, the next step in the study is focused on describing the influence of the edge roughness and thermal fluctuations when the finite Rashba field is taken into account. The effect of $\vec{H}_{R}$ with $\alpha_{R}=10^{-11} \mathrm{eVm}$ on the temporal evolution of $x(t)$ along the rough strip $\left(D_{g}=3 \mathrm{~nm}\right)$ under different $j_{a}$ is depicted in 


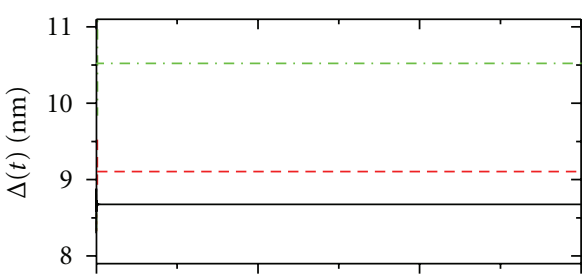

(a)

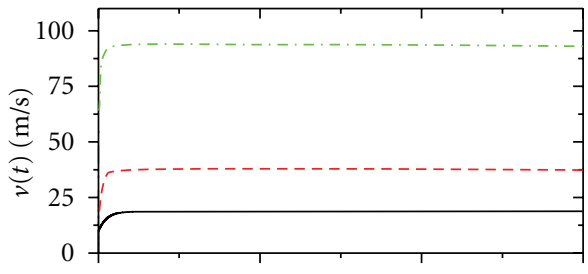

(b)

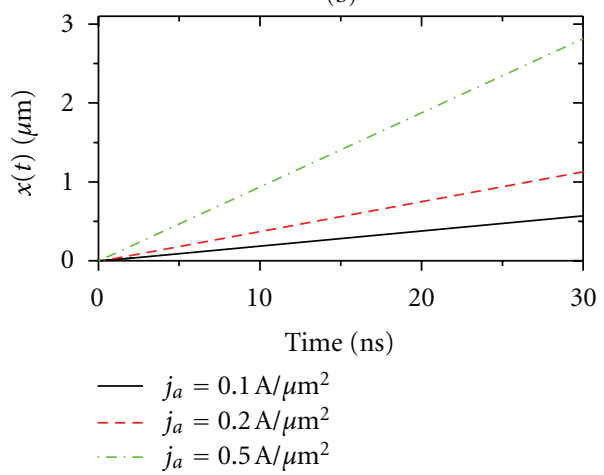

(c)

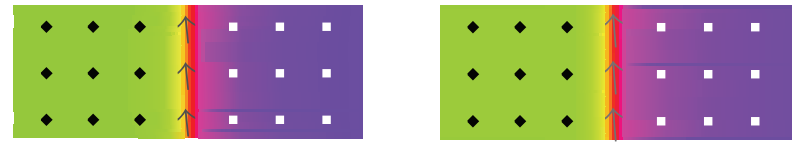

(d) $j_{a}=0.1 \mathrm{~A} / \mu \mathrm{m}^{2}$

$m_{z}=-1$

(e) $j_{a}=0.5 \mathrm{~A} / \mu \mathrm{m}^{2}$
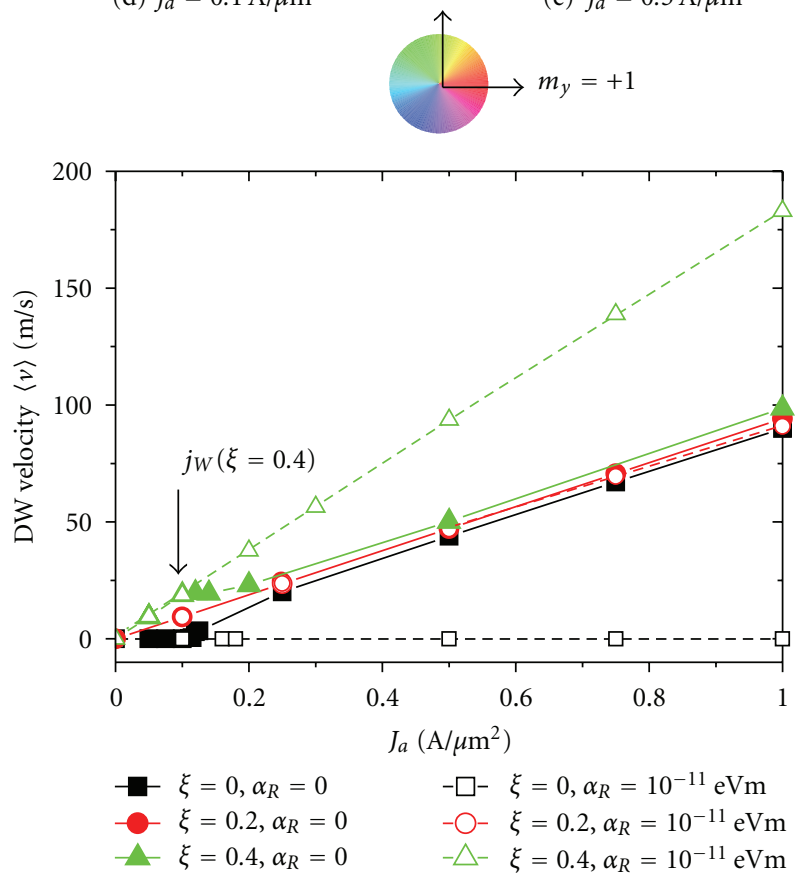

(f)

FIGURE 4: $\mu M$ results of the current driven DW dynamics along a perfect strip with $L_{y} \times L_{z}=120 \times 3 \mathrm{~nm}^{2}$ in the presence of SOI $\left(\alpha_{R}=\right.$ $\left.10^{-11} \mathrm{eVm}\right)$. (a), (b), and (c) depict the micromagnetically $(\mu M)$ computed temporal evolution of the DW width $\Delta$, the DW velocity $v$ and the DW position $x$ under three different density currents: $j_{a}=0.1 \mathrm{~A} / \mu \mathrm{m}^{2}, j_{a}=0.2 \mathrm{~A} / \mu \mathrm{m}^{2}$, and $j_{a}=0.5 \mathrm{~A} / \mu \mathrm{m}^{2}$ for a nonadiabatic parameter of $\xi=0.4$. (d)-(e) show typical micromagnetic states of the propagating DW in the presence of SOI. (f) Time-averaged DW velocity as function of $j_{a}$ for three different values of the nonadiabatic parameter: $\xi=0, \xi=0.2$, and $\xi=0.4$. Filled and open symbols correspond to the cases with $\alpha_{R}=0$ and $\alpha_{R}=10^{-11} \mathrm{eVm}$, respectivelly (Reprinted with permission From [72]. Copyright (2012), American Institute of Physics).

Figures $5(\mathrm{a})-5(\mathrm{c})$. A first quantitative difference with respect to the zero-Rashba field case $\left(\alpha_{R}=0\right)$ studied in Figure $3(\mathrm{~h})$ is that the deterministic depinning threshold current increases to $j_{d}=0.6 \mathrm{~A} / \mu \mathrm{m}^{2}$ in the presence of finite Rashba field with $\alpha_{R}=10^{-11} \mathrm{eVm}$. At zero temperature ( $T=0$, blue-dashed lines) two different behaviors are clearly observed. If $j_{a}$ is smaller than the deterministic threshold current $\left(j_{d}=0.6 \mathrm{~A} / \mu \mathrm{m}^{2}\right)$, the DW eventually departs from its initial position but after a few nanoseconds, it becomes totally pinned after reaching a region with high surface roughness (see Figures 5(a) and 5(b) for $j_{a}=0.2-$ $0.4 \mathrm{~A} / \mu \mathrm{m}^{2}$ ). On the other hand, for $j_{a} \geq j_{d}$, the DW position increases linearly as the time elapses in the whole evaluate temporal window $\left(t_{w}=50 \mathrm{~ns}\right)$.

The DW dynamics is substantial different at $T=300 \mathrm{~K}$. Ten stochastic realizations have been evaluated for each $j_{a}$. Even for very small currents (see red-solid lines in Figure 5(a)) there is a no-null probability of DW propagation. If $j_{a}$ increases below the critical deterministic threshold $\left(j_{a}<j_{d}\right)$, the DW propagates depicting a jerky motion as due to thermal activation over the local energy barrier induced by the roughness (see red-solid Figure 5(b)). The DW displaces several nanometers from its initial position during some time before reaching a region of high surface roughness, where it is temporally pinned up to thermal fluctuations assist again the DW depinning and its subsequent propagation. Similarly to the deterministic case, if $j_{a} \geq j_{d}$, the DW position increases almost linearly as the time elapses for all the realizations (see Figure 5(c)). Therefore, $j_{a}$ is high enough to overcome the energy barrier of the roughness independently on the thermal effects in such a high current regime. As it is depicted in Figures $5(\mathrm{~d})-5(\mathrm{~g})$, the DW Bloch structure is also preserved for all $j_{a}$ in the presence of the Rashba field at $T=300 \mathrm{~K}$.

The DW velocity as a function of $j_{a}$ is shown in Figure 5(h) for $\alpha_{R}=10^{-11} \mathrm{eVm}$. The time-averaged DW velocity $(\langle v\rangle)$ along a rough strip $\left(D_{g}=3 \mathrm{~nm}\right)$ at zero temperature ( $T=0$, blue-squares) are compared to the statistically-averaged DW velocity $([\langle v\rangle])$ at room temperature $(T=300 \mathrm{~K}$, red-circles), which was computed by averaging the time-averaged velocity over ten stochastic realizations for each $j_{a}$. Error bars indicate the standard 


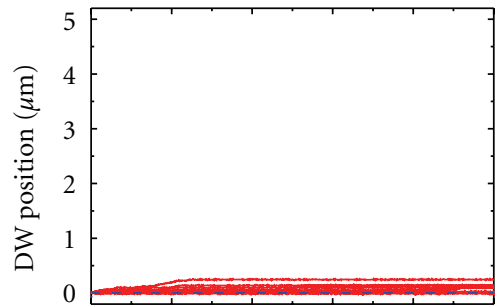

(a) $J_{a}=0.2 \mathrm{~A} / \mu \mathrm{m}^{2}$

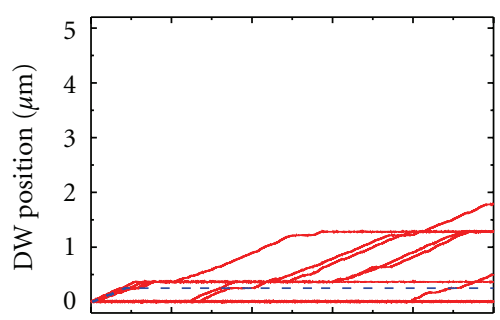

(b) $J_{a}=0.4 \mathrm{~A} / \mu \mathrm{m}^{2}$

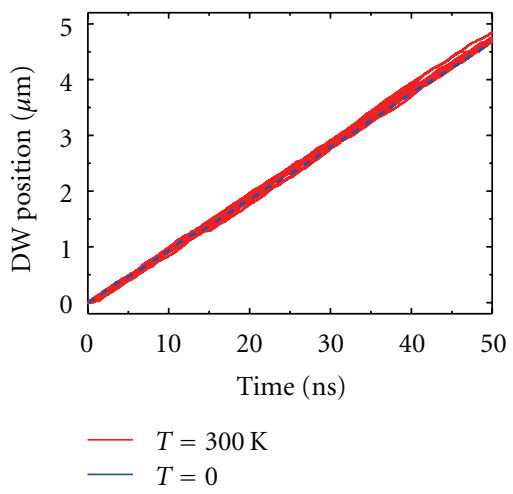

(c) $J_{a}=0.6 \mathrm{~A} / \mu \mathrm{m}^{2}$

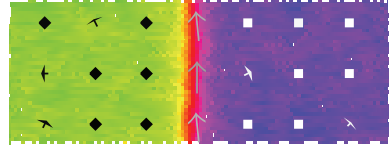

$J_{a}=0.1 \mathrm{~A} / \mu \mathrm{m}^{2}$

(d)

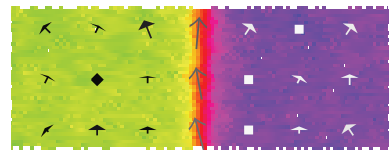

$J_{a}=0.6 \mathrm{~A} / \mu \mathrm{m}^{2}$

(f)

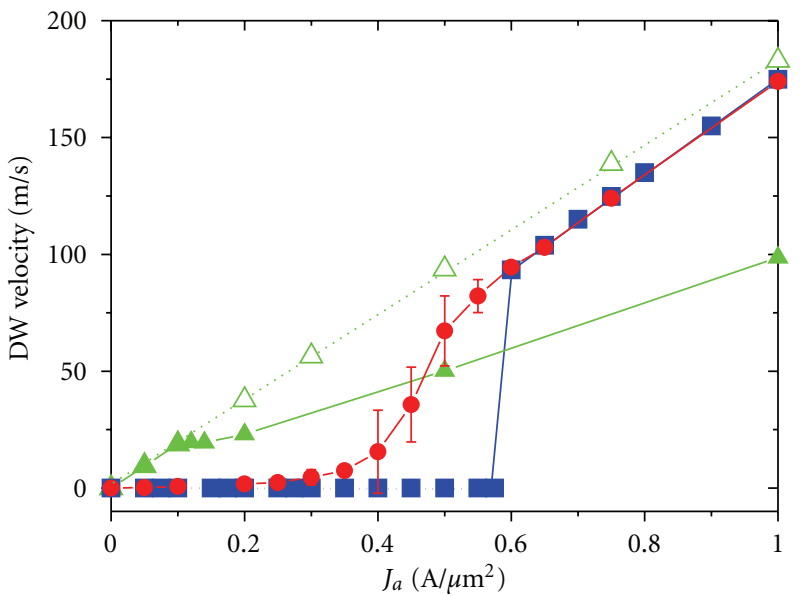

$\triangle D_{g}=0, \alpha_{R}=10^{-11} \mathrm{eVm}, T=0$

$\triangle D_{g}=0, \alpha_{R}=0, \mathrm{~T}=0$

- $D_{g}=3 \mathrm{~nm}, \alpha_{R}=10^{-11} \mathrm{eVm}, T=0$

- $D_{g}=3 \mathrm{~nm}, \alpha_{R}=10^{-11} \mathrm{eVm}, T=300 \mathrm{~K}$

(h)

Figure 5: (a)-(c) $x(t)$ versus $t$ along a rough strip $\left(D_{g}=3 \mathrm{~nm}\right.$ ) for different $j_{a}$ with $\alpha_{R}=10^{-11} \mathrm{eVm}$ at zero (blue-dashed lines) and at room temperature (red-solid lines). (d)-(g) Typical DW configurations under different $j_{a}$. (h) DW velocity as a function of $j_{a}$. (Reprinted with permission From [71]. Copyright (2012), American Institute of Physics).

deviation. The results for $\xi=0.4$ of former Figure $4(\mathrm{f})$ are also included for comparison. At $T=0$, no sustained DW motion is achieved up to $j_{a}$ overcomes the deterministic depinning threshold $j_{d}=0.6 \mathrm{~A} / \mu \mathrm{m}^{2}$, and above it $\left(j_{a} \geq j_{d}\right)$, the DW velocity increases linearly with $j_{a}$. The curve of $[\langle v\rangle]$ as a function of $j_{a}$ at $T=300 \mathrm{~K}$ is in good qualitative agreement with recent experiments (see Figure 3 in [47] and in [55]). One observes (i) a slow regime $\left(j_{a}<j_{d}\right)$ controlled by thermal activation and local pinning, where $[\langle v\rangle]$ increases exponentially, and (ii) a high-current flow regime $\left.\left(j_{a}\right\rangle j_{d}\right)$ with a linear variation of $[\langle v\rangle]$. Note that the DW mobility at the high-current regime $\left(j_{a}>j_{d}\right)$ is larger than the one achieved for $\vec{H}_{R}=0$ because the transverse Rashba field SOI avoids the turbulent DW precession.

Due to the high computational effort, these types of full micromagnetic studies of realistic strips with edge roughness at room temperature are very time-consuming, and in order to describe experimental results, it is desirable to develop the $1 \mathrm{DM}$ given by (5) and (6) by including the effect of the Rashba field given by (7). The resulting $1 \mathrm{DM}$ equations are

$$
\begin{aligned}
\left(1+\alpha^{2}\right) \frac{\dot{X}}{\Delta}= & \alpha \gamma_{0}\left(H_{e}+H_{p}(X)+H_{\mathrm{th}}(t)\right) \\
& +\frac{1}{2} \gamma_{0}\left(H_{K} \sin (2 \Phi)-\pi H_{R} \sin (\Phi)\right) \\
& -(1+\alpha \xi) \frac{1}{\Delta} \frac{\mu_{B} P}{e M_{s}} j_{a} \\
\left(1+\alpha^{2}\right) \dot{\Phi}= & \gamma_{0}\left(H_{e}+H_{p}(X)+H_{\mathrm{th}}(t)\right) \\
& -\alpha \frac{1}{2} \gamma_{0}\left(H_{K} \sin (2 \Phi)-\pi H_{R} \sin (\Phi)\right) \\
& -(\xi-\alpha) \frac{1}{\Delta} \frac{\mu_{B} P}{e M_{s}} j_{a},
\end{aligned}
$$




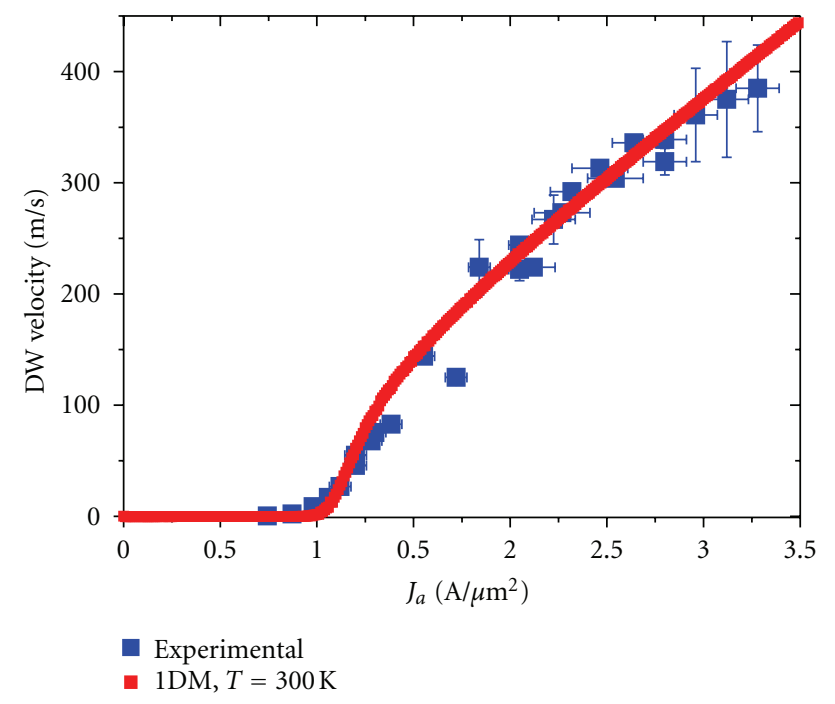

Figure 6: Comparison of the experimental results (see Figure 3 in [55]) and 1DM predictions for the current-induced DW dynamics along a Cobalt strip with high PMA sandwiched between two dissimilar nonmagnetic strips $(\mathrm{Pt} / \mathrm{Co} / \mathrm{AlO})$ at room temperature $(T=300 \mathrm{~K})$. All the $1 \mathrm{DM}$ parameters are enumerated in the text.

where $\vec{H}_{R}=H_{R} \vec{u}_{y}$ with $H_{R}=\alpha_{R} P j_{a} / \mu_{0} \mu_{B} M_{s}$. Although it is not shown here for reasons of briefness, it was confirmed that the $\mu \mathrm{M}$ results depicted in Figures 4(b), 4(c) and 4(f) are very accurately reproduced by these $1 \mathrm{DM}(8)$. Much more interesting is to show how this simple $1 \mathrm{DM}$ is able to reproduce the recent experimental results by Miron and coworkers [55]. In their experimental study, a cobalt strip with a crosssection of $L_{y} \times L_{z}=500 \mathrm{~nm} \times 0.6 \mathrm{~nm}$ is sandwiched between two dissimilar nonmagnetic layers $(\mathrm{Pt} / \mathrm{Co} / \mathrm{AlO})$. In order to reproduce these experimental results by using the $1 \mathrm{DM}(8)$, the following material parameters for the sandwiched Cobalt layer are considered: $M_{s}=1.09 \times 10^{6} \mathrm{~A} / \mathrm{m}, A=10^{-11} \mathrm{~J} / \mathrm{m}$, $K=1.19 \times 10^{6} \mathrm{~J} / \mathrm{m}^{3}, \alpha=0.2, P=0.5, \xi=1$, and $\alpha_{R}=$ $10^{-10} \mathrm{eVm}$. Note that these parameters are consistent with the ones which were used in $[55,79]$. The DW width is $\Delta=\sqrt{A / K}=3 \mathrm{~nm}, H_{K}=27852 \mathrm{~A} / \mathrm{m}$, and the effect of disorder is modeled by assuming a periodic pinning potential $V_{\text {pin }}(X)=V_{0} \sin ^{2}(\pi X / p)$ with a characteristic energy barrier of $V_{0}=1.8 \times 10^{-19} \mathrm{~J}$, and a periodicity of $p=30 \mathrm{~nm}$.

The $1 \mathrm{DM}$ results for the average DW velocity at room temperature are compared to the experimental results (see Figure 3 in [55]) in Figure 6. In the low current regime, the DW exhibits a stochastic creep motion, and the DW velocity can be described by $v=v_{0} \exp \left[-\left(j_{d} / j_{a}\right)^{\mu}\left(V_{0} / K_{B} T\right)\right]$ where $v_{0}$ is a prefactor, $j_{a}$ is the applied current, and $j_{d}$ is the critical depinning threshold. $V_{0}$ is the characteristic height of the pinning energy barrier induced by the surface roughness, and $\mu$ is a universal dynamics exponent. As in the field-driven case, the creep regime under current is consistent with an exponent of $\mu=1 / 4$. When the driving density current $j_{a}$ is well above the deterministic depinning threshold, thermal perturbations and surface roughness have a negligible effect on the DW velocity, which is found to increase linearly on $j_{a}$ similarly to the perfect strip case at zero temperature. As it clearly shown in Figure 6, the $1 \mathrm{DM}$ predictions are in very good agreement with the experimental measurements. This is a noticeable result, because a systematic experimental study of the DW velocity as function of the applied current along different strips with different materials, sizes, and configurations (single layer or asymmetric multilayer stacks) could be accurately reproduced by the $1 \mathrm{DM}$ simulations including both disorder and thermal effects, with low computational effort. By means of direct comparison with experimental measurements, it could be useful to gain information on the nonadiabatic parameter, for instance, by simply comparing with the high flow DW mobility. These type of comparative studies could be also used to extract the value of the Rashba parameter or the temperature dependence of the polarization factor. From a technological point of view, the SOI mediated by the Rashba field is a remarkable phenomenon because it promotes the high velocity and rigid DW propagation at relatively low current avoiding unwanted Joule heating.

\section{DW Depinning from a Notch}

The realization of DW-based devices for developing recording and logic technologies does not only require high velocity propagation with low current, but also an efficient control of the DW position. This can be done by means of constrictions or artificial notches which act as local pinning sites for the DW. The success of the applications require high stability against thermal fluctuations, and at the same time, lowcurrent DW depinning. This section is dedicated to the analysis of the pinning potential due to artificial notches intentionally designed to control the DW position in a strip with high PMA, and to the study of the DW depinnnig processes driven by both magnetic field and/or currents.

4.1. Describing the Pinning Potential. Figure 7(a) shows the geometry of strip containing an artificial pinning site, which consists on two rectangular notches, each one of dimensions $n_{x} \times n_{y}$ and placed at both sides of the strip. In the rest of this review, the parameters for a typical CoPtCr alloy are considered: $M_{s}=3 \times 10^{5} \mathrm{~A} / \mathrm{m}, A=10^{-11} \mathrm{~J} / \mathrm{m}, K=2 \times$ $10^{5} \mathrm{~J} / \mathrm{m}^{3}, P=0.5, \alpha=0.2$, and $\alpha_{R}=0$, and the crosssection of the strip is fixed to $L_{y} \times L_{z}=60 \mathrm{~nm} \times 3 \mathrm{~nm}$. Figure 7(b) depicts the pinned equilibrium state of a Bloch DW at rest for a pinning site with $n_{x}=15 \mathrm{~nm}$ and $n_{y}=6 \mathrm{~nm}$. In order to describe the pinning potential $V_{\text {pin }}$ induced by the constriction, the temporal evolution of the DW position was micromagnetically computed under static fields $\vec{B}_{e}=$ $B_{e} \vec{u}_{z}$ along the easy $z$-axis for three different widths $\left(n_{y}\right.$ : $3 \mathrm{~nm}, 6 \mathrm{~nm}, 9 \mathrm{~nm})$ and fixed length $\left(n_{x}=15 \mathrm{~nm}\right)$. After a few damped oscillations (not shown), the DW reaches final equilibrium position $X_{\mathrm{eq}}$ if the applied field is smaller than the depinning threshold $\left(B_{e}<B_{d}\right)$, which depends on the length $\left(n_{x}\right)$ and the width $\left(n_{y}\right)$ of the notch. Figure $7(\mathrm{c})$ indicates that, except for fields close to the de-pin-ning threshold, the equilibrium DW position increases from the center of the constriction almost linearly with the applied field. The slope 


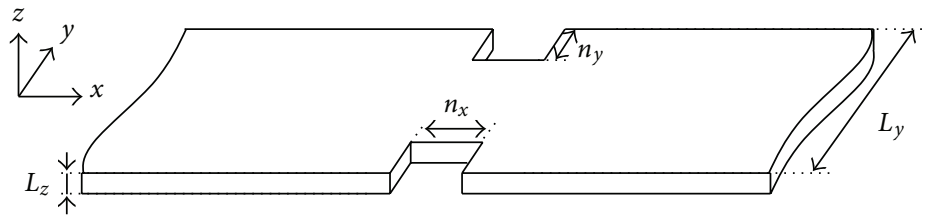

(a)

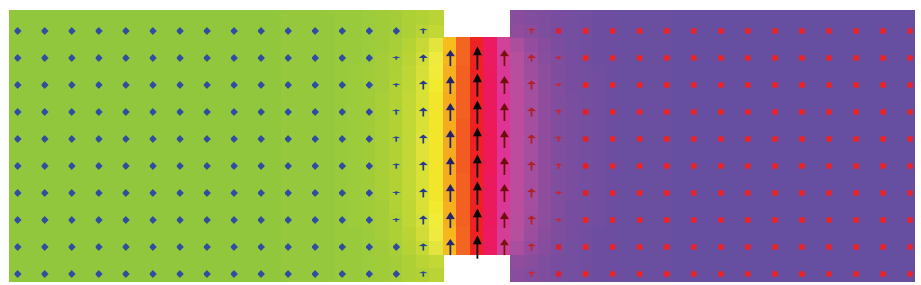

(b)

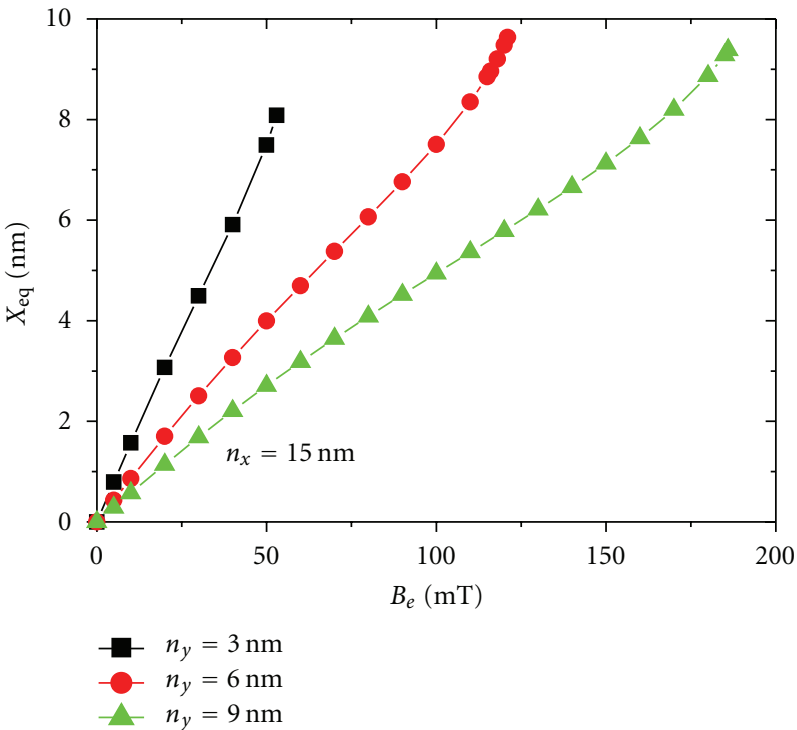

(c)

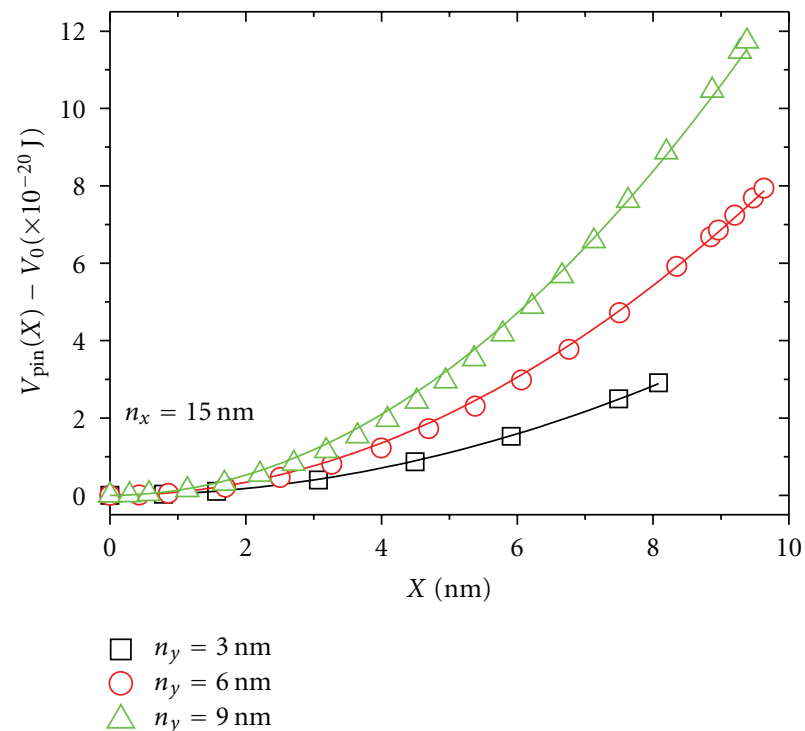

(d)

Figure 7: (a) Scheme of a pinning site consisting on two rectangular notches at both edges of the strip. (b) Equilibrium state of a pinned DW in a CoPtCr strip of $L_{y} \times L_{z}=60 \mathrm{~nm} \times 3 \mathrm{~nm}$ with a constriction described by $n_{x}=15 \mathrm{~nm}$ and $n_{y}=6 \mathrm{~nm}$. (c) Equilibrium DW position as a function of the applied field $B_{e}$ in the pinned regime. (d) Pinning potential $V_{\text {pin }}$ as a function of the DW position. Dots correspond to micromagnetic results and the solid lines are the fittings to $V_{\text {pin }}(X)=(1 / 2) K_{N} X^{2}$. In these last graphs the length of the notch is fixed to $n_{x}=15 \mathrm{~nm}$, and three different widths are evaluated: $n_{y}: 3 \mathrm{~nm}, 6 \mathrm{~nm}, 9 \mathrm{~nm}$.

of this increasing decreases with $n_{y}$. The pinning potential depicted in Figure 7(d) was computed from the total energy by subtracting the Zeemann contribution for each state [29]. In this pinned regime, the pinning potential can be fitted to a parabolic profile given by $V_{\text {pin }}(X)=(1 / 2) K_{N} X^{2}$ for $|X| \leq L_{N} . K_{N}$ is the elastic constant of the constriction, and $L_{N}$ is the half length of the pinning potential. From $\mu M$ results of Figure $7(d)$, the following values are deduced for a constriction with $n_{x}=15 \mathrm{~nm}$ and $n_{y}=6 \mathrm{~nm}: K_{N}=$ $1.7 \times 10^{-3} \mathrm{~N} / \mathrm{m}$ and $L_{N}=9.5 \mathrm{~nm}$.

4.2. Field and Current-Driven DW Depinning. Once described the pinning potential, let us analyze the field and current DW depinning, in particular for a strip with $L_{y} \times$ $L_{z}=60 \mathrm{~nm} \times 3 \mathrm{~nm}$ with a constriction characterized by $n_{x}=15 \mathrm{~nm}$ and $n_{y}=6 \mathrm{~nm}$. The goal is to evaluate how the depinning field $B_{d}$ depends on the applied current $j_{a}$ for several values of the nonadiabatic parameter $\xi$, firstly at zero temperature. The problem has been studied from both micromagnetic simulations $(\mu M)$ and one-dimensional model (1DM). In the case of the $1 \mathrm{DM}$, the DW width was obtained from equilibrium state of the pinned DW $(\Delta=8.25 \mathrm{~nm})$, and the hard-axis anisotropy field $\left(H_{K}\right)$ of magnetostatic origin was deduced from the micromagnetically computed Walker breakdown field $\left(B_{W}=\mu_{0} H_{W} \approx\right.$ $0.33 \mathrm{mT}$ ), which results in $H_{K}=2 H_{W} / \alpha=2626.06 \mathrm{~A} / \mathrm{m}$. The pinning potential is given by $V_{\text {pin }}(X)=(1 / 2) K_{N} X^{2}$ for $|X| \leq$ $L_{N}$ with $K_{N}=1.7 \times 10^{-3} \mathrm{~N} / \mathrm{m}$ and $L_{N}=9.5 \mathrm{~nm}\left(V_{\text {pin }}(X)=\right.$ 0 for $\left.|X| \geq L_{N}\right)$. The results are depicted in Figure 8(a) for several values of the nonadiabatic parameter $\xi$. A good 


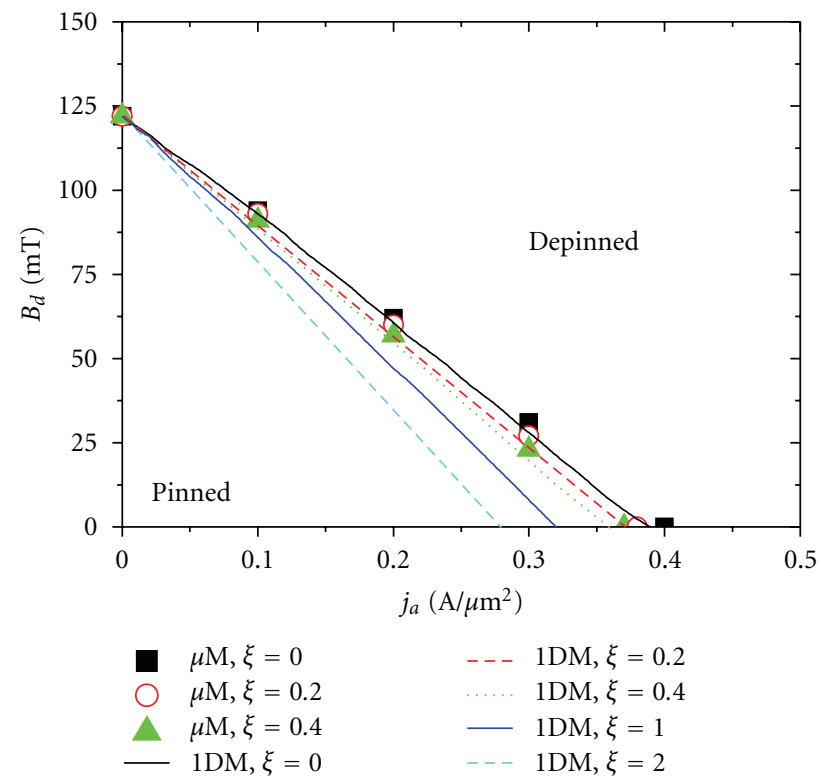

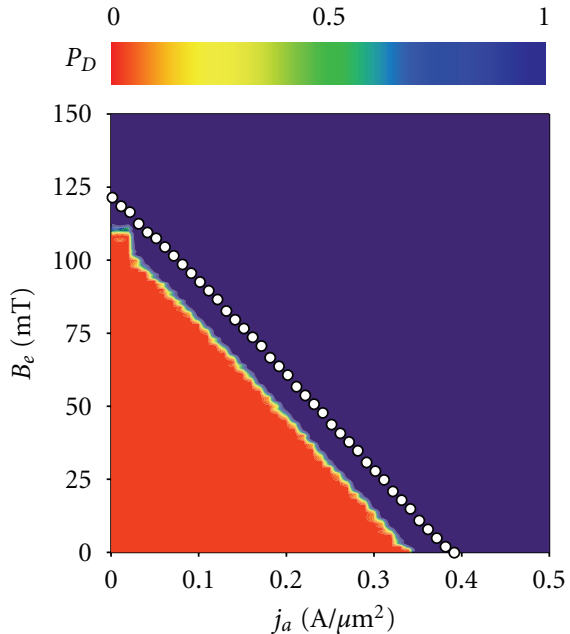

(b)

(a)

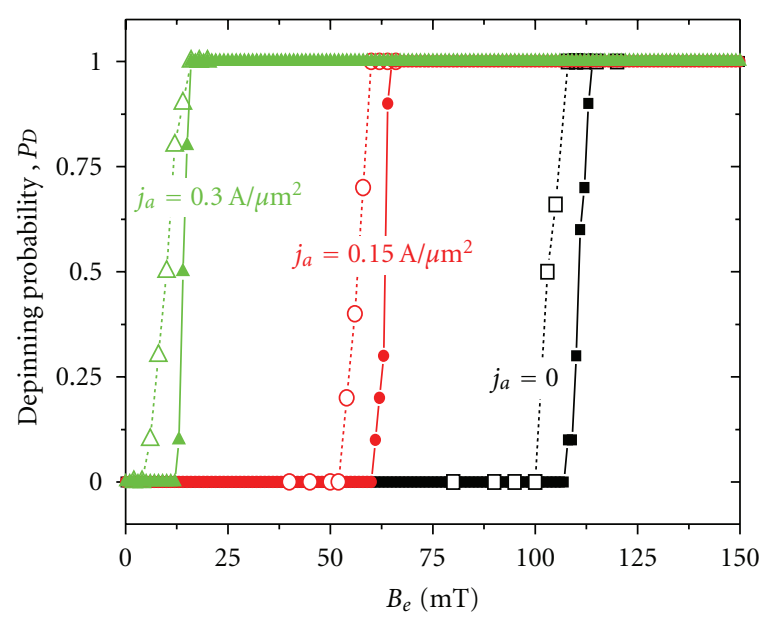

(c)

Figure 8: (a) Depinnig field $B_{d}$ as a function of $j_{a}$ for several values of $\xi$ for a CoPtCr strip with $L_{y} \times L_{z}=60 \mathrm{~nm} \times 3 \mathrm{~nm}$ and $n_{x}=15 \mathrm{~nm}$ and $n_{y}=6 \mathrm{~nm}$. Dots correspond to $\mu M$ results and lines correspond to the ones obtained from the $1 \mathrm{DM}$ with $\Delta=8.25 \mathrm{~nm}, H_{K}=2626.06 \mathrm{~A} / \mathrm{m}$, and a pinning potential given by $V_{\text {pin }}(X)=(1 / 2) K_{N} X^{2}$ for $|X| \leq L_{N}$ with $K_{N}=1.7 \times 10^{-3} \mathrm{~N} / \mathrm{m}$ and $L_{N}=9.5 \mathrm{~nm}\left(V_{\text {pin }}(X)=0\right.$ for $\left.|X| \geq L_{N}\right)$. (b) Probability of DW depinning $\left(P_{D}\right)$ as a function of both $\left(B_{e}, j_{a}\right)$ obtained from $1 \mathrm{DM}$ with $\xi=0$ at $T=300 \mathrm{~K}$. A temporal window of $t_{w}=500 \mathrm{~ns}$ was considered, and 100 stochastic realizations were evaluated. Open dots represent the deterministic $(T=0)$ depinning field $B_{d}\left(j_{a}\right)$. (c) Examples of $P_{D}$ as a function of $B_{e}$ under three different density currents: $j_{a}=0$ (squares), $j_{a}=0.15 \mathrm{~A} / \mu \mathrm{m}^{2}($ circles) and $j_{a}=0.3 \mathrm{~A} / \mu \mathrm{m}^{2}$ (triangles). Filled and open symbols correspond to $1 \mathrm{DM}$ and $\mu M$ results respectively (Reprinted with permission From [65]. Copyright (2012), American Institute of Physics).

quantitative agreement is observed between micromagnetic results (dots) and $1 \mathrm{DM}$ predictions (lines). The depinning field $B_{d}\left(j_{a}, \xi\right)$ decreases linearly with the applied current $j_{a}$, and the diminution of $B_{d}$ as a function of $j_{a}$ becomes slightly stronger as the nonadiabatic parameter increases, but the linear behavior is preserved for all analysed values.

The results of Figure 8(a) were obtained at zero temperature $(T=0)$. In order to determine whether thermal fluctuations are likely to play an important role in the DW depinning process, the probability of DW depinning $\left(P_{D}\right)$ has been studied at $T=300 \mathrm{~K}$ by numerically solving the $1 \mathrm{DM}$ (5) and (6). A temporal window of $t_{w}=500 \mathrm{~ns}$ was considered, and in order to present statistically meaningful results, 100 stochastic realizations were evaluated for each pair of $\left(B_{e}, j_{a}\right)$. The results for $\xi=0$ are depicted in Figure $8(\mathrm{~b})$, where open dots correspond to the deterministic $(T=0)$ depinning threshold. At $T=0$, the DW depinning only occurs if the current and the field are sufficiently large. On the contrary, at $T=300 \mathrm{~K}$, the problem is no longer deterministic, and there is a non-null probability 
of DW depinning for fields and currents smaller than the deterministic threshold. As it is observed, thermal fluctuations significantly reduces the depinning field $B_{d}$ under a given current with respect to deterministic case. For instance, under zero current $\left(j_{a}=0\right)$, the depinning field at $T=$ 0 is $\approx 123 \mathrm{mT}$, whereas the probability of DW depinning becomes $100 \%$ with a minimum field of $114 \mathrm{mT}$ at room temperature. As it is shown in both Figures 8(b) and 8(c), the probability of DW depinning changes from $0 \%$ to $100 \%$ in a reduced range of fields, for example, from $60 \mathrm{mT}$ to $65 \mathrm{mT}$ under a current of $j_{a}=0.15 \mathrm{~A} / \mu \mathrm{m}^{2}$ (see filled red circles in Figure 8(c)).

A similar analysis of thermal effects at $T=300 \mathrm{~K}$ was also carried by means of full micromagnetic modeling $(\mu M$, (1)) in the perfect adiabatic case. The probability of DW depinning $\left(P_{D}\right)$ micromagnetically computed as a function of $B_{e}$ is shown in Figure 8(c) by means of open symbols. Similarly to one-dimensional results, the transition from $P_{D}=0$ to $P_{D}=1$ is gradual and takes place in a narrow range of fields, but it is slightly anticipated toward smaller fields because the nonuniformities in the local magnetization assist the thermal depinning.

In summary, as it is desirable for memory applications, the field required to promote the DW depinning in absence of current is very high because the energy barrier required to overcome the pinning potential is around 19 times larger than the thermal energy at room temperature for a typical strip as the one studied here. The depinning field decreases linearly as the current is raised, and the slope of this declension slightly increases with the nonadiabaticity. Under zero field in the perfect adiabatic limit, the critical depinning density current is around $0.4 \mathrm{~A} / \mu \mathrm{m}^{2}$ at zero temperature. This corresponds to a threshold current of $72 \mu \mathrm{A}$, which is small enough to minimize unwanted Joule heating effects. It was found that thermal fluctuations at room temperature play a significant role on the DW depinning by reducing the depinning field for a given current with respect to the deterministic case. These results are in good qualitative agreement with the experimental measurements by Ravelosona el al. [44] exploring the high pinning regime $\left(B_{d} \gg \mu_{0} H_{K} / \alpha\right)$, and they explain the linear decreasing of the depinning current with increasing field. In that experiment [44], the depinning current at zero field was found around $0.1 \mathrm{~A} / \mu \mathrm{m}^{2}$, which, in spite of the different materials and geometry, is in the same order of magnitude than our results. The Joule heating effect was estimated less than $20 \mathrm{~K}$ at the highest current densities applied, which is small enough to justify a Lagenvin description with constant temperature. By simulating the same geometry and materials than these experiments, the stochastic one-dimensional model can be directly adopted in order to gain a better description of the experimental observations by taking into account the effect of the constant temperature of the sample, and, therefore, a more accurate estimate of both the polarization factor and the nonadiabatic parameter, along with their dependence on the temperature could be addressed. On the other hand, the presented analysis also shows that the probability of the DW depinning under a given current changes abruptly from $0 \%$ to $100 \%$ in a narrow range of fields. This result points out that the DW depinning is highly selective, which is also relevant for further technological applications. All these theoretical predictions indicate that engineering of pinning sites in thin strips of high perpendicular anisotropy provides an efficient pathway to achieve both high stability against thermal fluctuations, and at the same time, low-currentinduced domain wall depinning.

\section{DW-Based Nano-Oscillators}

The theoretical studies of the current-driven DW dynamics along a strip with high PMA, and its pinning and depinning driven by fields and/or current, are relevant for further designing recording and logic devices based on DWs. However, these are not the only potential applications of DWs, which could be also useful in other branches of the nanotechnology. This last section is dedicated to the numerical study of the pinned DW oscillations driven by static currents, which could find application to develop novel DW-based nanoscillators.

5.1. Pinned DW Oscillations. Let us focus our attention on a CoPtCr strip $\left(M_{s}=3 \times 10^{5} \mathrm{~A} / \mathrm{m}, A=10^{-11} \mathrm{~J} / \mathrm{m}, K=\right.$ $2 \times 10^{5} \mathrm{~J} / \mathrm{m}^{3}, \alpha=0.2$, and $\left.P=0.4\right)$ with $L_{y} \times L_{z}=$ $60 \times 3 \mathrm{~nm}^{2}$ containing a single pinning site which consists on two rectangular notches $\left(n_{x}=15 \mathrm{~nm}\right.$ long, $n_{y}=6 \mathrm{~nm}$ wide) symmetrically placed at both edges of the strip. The micromagnetically computed temporal evolution of the DW position $X(t)$ in the pinned regime $\left(j_{a}<j_{d}\left(\xi=0, B_{e}=0\right)=\right.$ $\left.0.4 \mathrm{~A} / \mu \mathrm{m}^{2}\right)$ is depicted in Figure $9(\mathrm{a})$ for three values of the applied current $j_{a}$ in the perfect adiabatic case $(\xi=0)$. For current smaller than Walker threshold $\left(j_{a}<j_{W}(0)\right)$, the DW moves during the first nanoseconds, but after that it returns to its initial state where it finally rests. If the applied current surpasses the Walker value $\left(j_{a} \geq j_{W}(0)=0.025 \mathrm{~A} / \mu \mathrm{m}^{2}\right)$, and at the same time, it remains smaller than the critical depinning threshold $\left(j_{a}<j_{d}\left(\xi=0, B_{e}=0\right)=0.4 \mathrm{~A} / \mu \mathrm{m}^{2}\right)$, the DW develops pinned oscillations periodically rotating between Bloch and Neel configurations. Examples of these DW configurations are depicted in Figures 9(b)-9(e) in the adiabatic case for $j_{a}=0.05 \mathrm{~A} / \mu \mathrm{m}^{2}$. Under such lowdensity currents in the pinned regime (see Figures 9(b)$9(\mathrm{e}))$, the DW magnetization oscillates around the $z$-axis symmetrically with respect to the center of the notch, whereas under high density currents in the pinned regime (not shown) the central position of DW oscillations is pushed to the right-hand side due to the spin torque $[40,41]$. The DW position $X_{\mu M}$ has two contributions: a static one $X_{\mathrm{dc}}$, which represents the central position around which the DW oscillates, and a periodically oscillating contribution at a frequency $f$ with an amplitude $X_{\mathrm{ac}}$. The dependence of these three variables on the applied density current $j_{a}$ is shown Figures 9(f), 9(g), and 9(h), respectively, for several values of the nonadiabatic parameter $(0 \leq \xi \leq 2 \alpha)$. Both $X_{\mathrm{dc}}$ and $f$ increase almost linearly with $j_{a}$, but the amplitude of the DW oscillations $X_{\mathrm{ac}}$ decreases from the maximum value reached just above the Walker current. Except for a slight reduction of the critical depinning current $j_{\operatorname{dep}}\left(\xi, B_{e}=0\right)$, it is clear that 


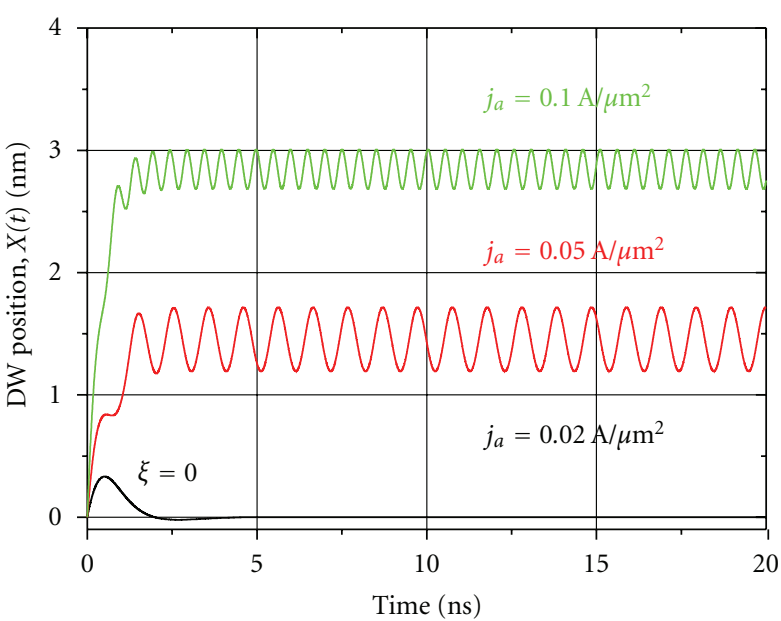

(a)

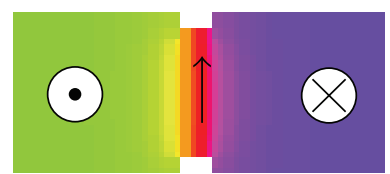

(b)

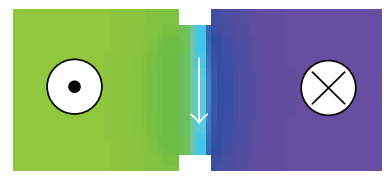

(d)

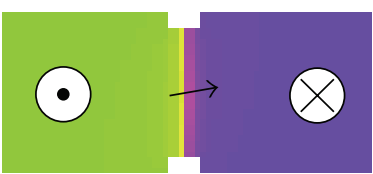

(c)

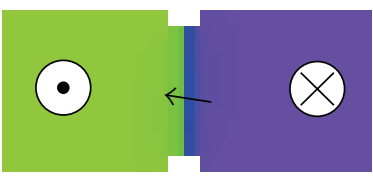

(e)

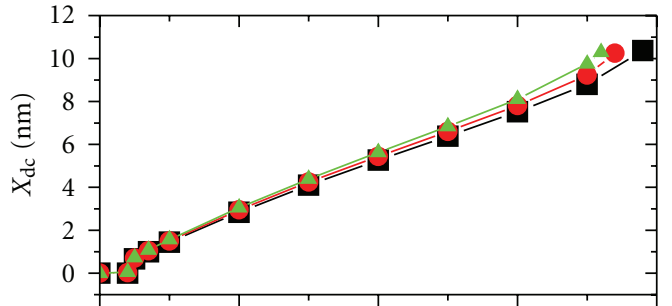

(f)

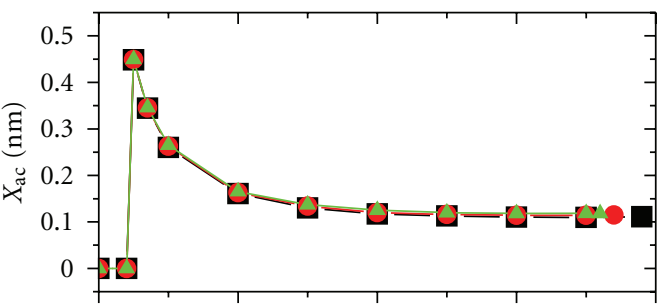

(g)

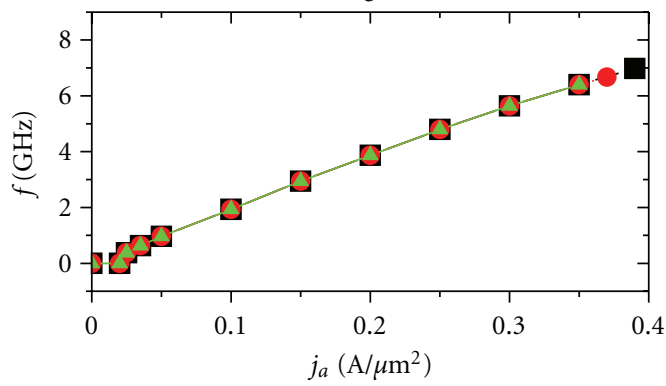

$-\xi=0$
$-\xi=0.2$
$-\xi=0.4$

FIGURE 9: $\mu M$ results for the DW dynamics under dcs in the pinned regime. The cross-section is $L_{y} \times L_{z}=60 \mathrm{~nm} \times 3 \mathrm{~nm}$, and the dimensions of each rectangular notch are $n_{x}=15 \mathrm{~nm}$ and $n_{y}=6 \mathrm{~nm}$. (a) DW position $X(t)$ as a function of time under dcs in the perfect adiabatic case $(\xi=0)$. (b)-(e) depict the Bloch and Neel pinned DW configurations under $j_{a}=0.05 \mathrm{~A} / \mu \mathrm{m}^{2}$. (f) Central DW position $\left(X_{\mathrm{dc}}\right)$, (g) Amplitude $\left(X_{\mathrm{ac}}\right)$, and (h) frequency $f$ of DW oscillations as a function of $j_{a}$ in the pinned regime. (From [41]).

the results do not depend significantly on the nonadiabaticity $\xi$. Although they are not shown here, it was also verified in [41] that the $1 \mathrm{DM}$ results for the pinned DW oscillations are similar to the $\mu M$ ones collected in Figures 9(a), 9(f)- 9(h).

It is worthy to note that the complete analysis of the frequency of the localized DW oscillation requires either perform full micromagnetic simulations (as in Figure 9) or numerical solving the complete $1 \mathrm{DM}$ (5) and (6) (as it was done in [41]). For instance, note from Figures $9(\mathrm{f})$ and $9(\mathrm{~g})$ that although the time average of the DW velocity is zero, its instantaneous value is not exactly null. However, it is also possible to obtain some information from these $1 \mathrm{DM}$ (5) and (6), which can be simplified by assuming some justified approximations. In particular, as the DW oscillations take place in the absence of driving field $\left(H_{e}=0\right)$ when the shape anisotropy is avoided $\left(H_{K}=0\right)$, and as the DW velocity is zero averaged over a period of the pinned oscillations $(\dot{X} \approx$ $\langle\dot{X}\rangle=0)$, it is possible to extract from (5) an expression for the pinning field $\gamma_{0} H_{p}(X)=((1+\xi \alpha) / \Delta)\left(\mu_{B} P / e M_{s}\right) j_{a}$. Introducing this result in (6), we reach to

$$
\dot{\Phi}=-\frac{1}{\alpha \Delta} \frac{\mu_{B} P}{e M_{s}} j_{a}
$$

so the frequency of the pinned DW oscillations is $f=\dot{\Phi} / \pi$ which is independent of the nonadiabatic parameter and increases linearly with $j_{a}$. This result is good quantitative agreement with the full micromagnetic results of Figure 9(f). Other $1 \mathrm{DM}$ results and further micromagnetic details, such as the dependence of the pinned regime with the dimensions of the notches, can be seen in our former work [41].

5.2. Spin Pumping and Induced Voltage Signal. Results of former Figure 9 indicate that it is possible to achieve pinned DW oscillations just driven by de via spin transfer torque. As electrons flow through the ferromagnetic strip, their spins tend to align with the magnetization. When they pass into the nonuniform magnetization region occupied by the DW, the electron spins rotate to stay aligned with the local magnetization. A reaction torque on the changing magnetization in the DW cause the pattern of magnetization at the DW to move in the direction of the electron flow. Considering perfect adiabatic conditions, the DW will move along a perfect strip if the applied density current is larger than the Walker breakdown $\left(j_{a}>j_{W}(0)\right)$, and in its displacement, the DW experiences periodic transformations 
between Bloch and Neel configurations. If the DW is trapped at a constriction, the spin transfer torque can promote pinned DW oscillations provided that the applied density current is above the Walker breakdown and below the critical depinning current $\left(j_{W}(0)<j_{a}<j_{d}\right)$. The complementary effect, spin pumping [82], occurs when the pinned DW oscillation drives a spin current along the strip, which results in an induced voltage difference between the ends of the strip.

The rest of this section is dedicated to the analysis of the voltage signal produced by these pinned DW oscillations. It has been theoretically predicted that magnetization dynamics induces an electromotive and spin-motive force (emf/smf), which acts on the conduction electrons through the spin Berry phase [39, 83-87], and, therefore, magnetization dynamics can be a source of voltage signal for external circuits. Berger [39] provided the first theoretical prediction of this effect in terms of an analog of the Josephson effect: a static electric current larger than the Walker breakdown threshold can promote the DW rotation at a given frequency $f=(1 / \pi) \dot{\Phi}$. He also predicted that the DW oscillation gives rise to a voltage signal given by $V_{\text {smf }}=-(\hbar P / 2 e) \dot{\Phi}$. Barnes and Maekawa [85] analyzed the generation of spin and charge current by a moving DW via electromotive forces considering the case that the spin is fully conserved. More recently, Duine $[82,88]$ pointed out that the voltage signal will be augmented by a nonadiabatic contribution, so it will be given by $V_{\mathrm{smf}}=-(\hbar P / 2 e)(\dot{\Phi}-\xi(\dot{X} / \Delta))$ in the $1 \mathrm{DM}$ approach. The first experimental observation of the electromotive force induced by a moving DW was carried out recently by Yang and coworkers [89], where external fields larger than the Walker breakdown were used to drive a DW along a soft Permalloy strip, and the averaged induced voltage produced by its DW transformation between transverse and vortex configurations was $V_{\mathrm{smf}}=-(\hbar \mathrm{P} / e)\left(\gamma_{0} H_{e}\right)$, where $H_{e}>H_{W}$.

Early theoretical predictions $[39,82,85,86,88]$ for the voltage induced by DW oscillations considered an idealized rigid DW, where the DW width $\Delta$ remains fixed. However, as it was confirmed in Figure 2(a), the DW width changes above the Walker breakdown $(\Delta=\Delta(t))$, and these changes could play significant role in the induced voltage signal. Here, we will focus on describing the induced voltage signal due to the pinned DW oscillations driven by dc in a hard PMA strip from a full micromagnetic point of view, which straightforwardly accounts for the internal complexities of the dynamics changes of DW width. The effective electric field induced by a time-dependent magnetic texture is computed according to the formalism developed by Tserkovnyak and Mecklenburg [87]. The component of this field along the length of the strip ( $x$-axis) is given by [87]

$$
E_{\mathrm{smf}, x}=-\frac{\hbar P}{2 e}\left[\vec{m} \cdot\left(\frac{\partial \vec{m}}{\partial t} \times \frac{\partial \vec{m}}{\partial x}\right)+\xi\left(\frac{\partial \vec{m}}{\partial t} \cdot \frac{\partial \vec{m}}{\partial x}\right)\right] .
$$

The first term at the right hand side of (10) is the perfect adiabatic contribution to the electric field induced by a time-dependent magnetization texture, which was derived by considering that the exchange field provided by the localized magnetic $d$ orbitals (local $\vec{m}$ ) is larger than the spin-diffusion
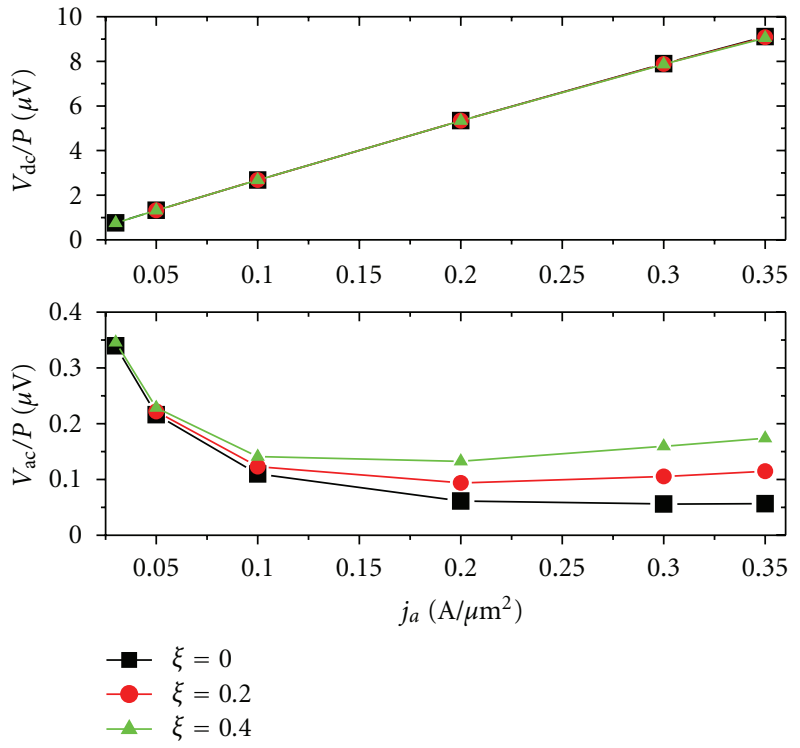

FIGURE 10: Voltage signal as due to the pinned DW oscillations driven dcs as computed from $\mu M$ simulations. Static $\left(V_{\mathrm{dc}}\right)$ and oscillating $\left(V_{\mathrm{ac}}\right)$ contributions of the voltage signal $V_{\text {smf }}(t)$ are depicted in (top) and (bottom), respectively. (from [41]).

length, and, therefore, assumes that the spin of conductions electrons perfectly align with local magnetization $[39,86]$. It is equally direct to interpret the first contribution to (10) in terms of the rate of the Berry-phase accumulation by spins adiabatically following the steady exchange field $[85,89]$. The second term at the right hand side of (10) is proportional to the nonadiabatic parameter $\xi$. This correction is related to slight spin misalignment of electron propagating through an inhomogeneous magnetic texture with the local magnetization [87]. In the limit of infinite exchange field this misalignment vanished (and so should $\xi)$, and only the adiabatic contributions would remain. The nonadiabatic corrections to the induced electric field by a time-dependent magnetic texture was firstly proposed by Duine [82] for a rigid DW. This nonadiabatic contribution can be viewed as a correction to the topological structure of the electron transport rigidly projected on the local magnetization texture. A more detailed description of the microscopic origin of (10) is far from the scope of this work, and they can be consulted in $[39,82,85-88]$. Once evaluated (10), the induced voltage between the ends of the strip is obtained by integrating over the strip length $\left(L_{x}=1.2 \mu \mathrm{m}\right)$ as $V_{\mathrm{smf}}=-\int_{0}^{L_{x}} E_{\mathrm{smf}, x} d x$.

The $\mu M$ results of the induced voltage signal $V_{\text {smf }}$ corresponding to the pinned DW oscillations described in former Figure 9 are depicted in Figure 10. They show that the induced voltage $V_{\text {smf }}(t)$ has also two contributions: a static one $V_{\mathrm{dc}}$ which increases linearly with $j_{a}$ independently on the nonadiabatic parameter $\xi$ (Figure 9(a)), and a periodic contribution with amplitude $V_{\mathrm{ac}}$ (see Figure 10(b)) oscillating at the same frequency $f$ as the DW position oscillations of Figure 9(h). An interesting point from a fundamental point 


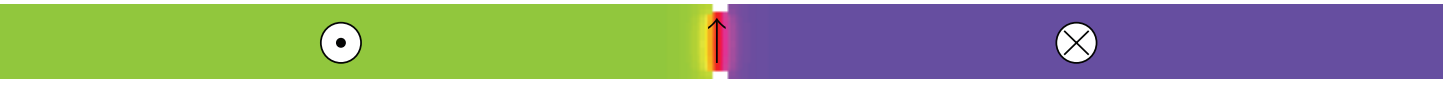

(a)

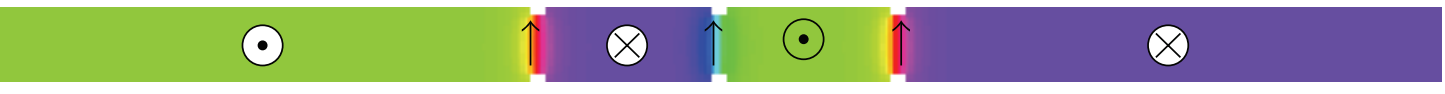

(b)

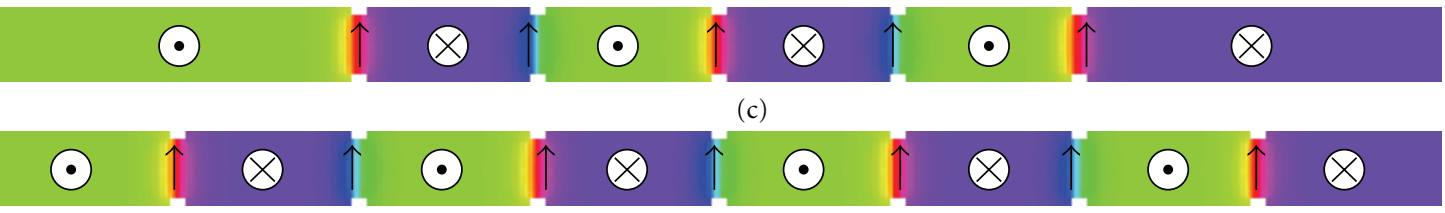

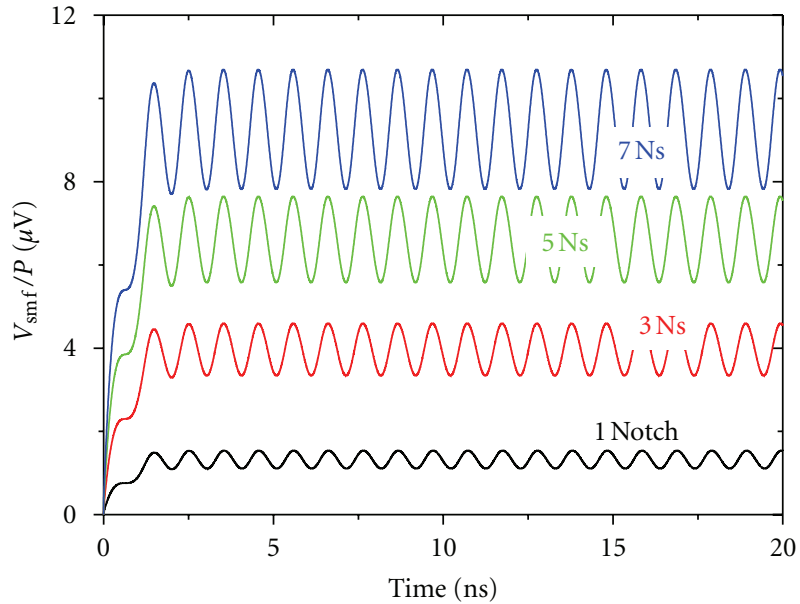

(e)

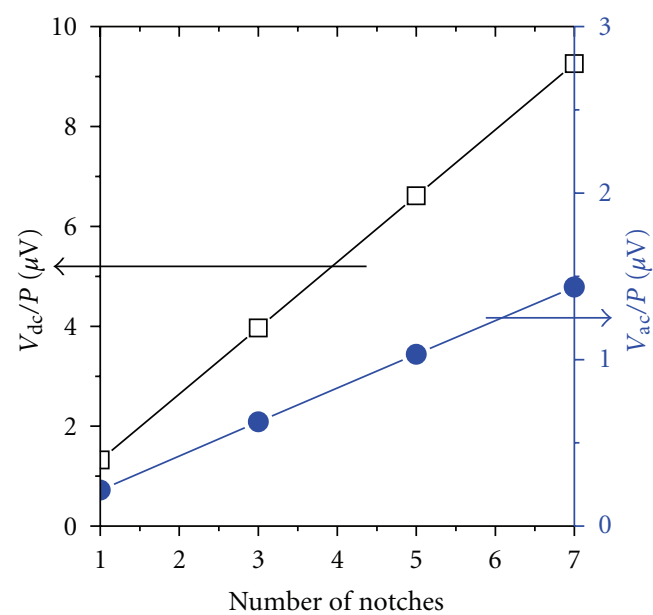

(f)

FIGURE 11: Equilibrium states with (a) one, (b) three, (c) five, and (d) seven Bloch DWs pinned at different notches separated each other by $S=138 \mathrm{~nm}$. (e) Voltage signal as a function of time for a strip with different number of pinned DWs. The applied current is $j_{a}=0.05 \mathrm{~A} / \mu \mathrm{m}^{2}$ and the perfect adiabatic case was considered. (f) Static $\left(V_{\mathrm{dc}}\right)$ and oscillating $\left(V_{\mathrm{ac}}\right)$ contributions of the voltage signal $V_{\text {smf }}(t)$ as a function of the number of pinned DWs. (from [41]).

of view is that, contrary to the $V_{\mathrm{dc}}$ case, the amplitude of the oscillating contribution $V_{\text {ac }}$ shows a dependence on the nonadiabatic parameter $\xi$, and therefore, if this signal can be experimentally detected, it could give information on the nonadiabaticity of system.

5.3. Enhancing the Induced Voltage Signal. From a technological point of view, the voltage signals induced by the pinned DW oscillations might prove useful to design nanoscale microwave oscillators and generators, which could find application in several fields such as sensors, telecommunications, or rf assisted writing for memory devices. However, for a given strip with a given constriction the amplitude of the dc voltage signal $V_{\mathrm{dc}}$ is restricted to the values of the $\mathrm{dc}$ applied current densities of the oscillating pinned regime $\left(j_{W}<j_{a}<j_{\text {dep }}\right)$. Moreover, the amplitude of the oscillating contribution $V_{\text {ac }}$ decreases with $j_{a}$ from $j_{W}$ to $j_{\text {dep }}$, and it will be desirable to enhance and extend the operation regime towards higher values of both $V_{\mathrm{dc}}$ and $V_{\mathrm{ac}}$ with the aim of fulfilling further technological requirements. In order to do it, we proposed to introduce several pinned DWs along the strip [41].
Figure 11 shows the equilibrium state of a CoPtCr strip containing (a) one, (b) three, (c) five, and (d) seven Bloch DWs separated each other from $S=138 \mathrm{~nm}$. The length and the cross-section of the strip, along with the shape and dimensions of each constriction are the same than in previous subsections: $L_{x}=1.2 \mu \mathrm{m}, L_{y}=60 \mathrm{~nm}, L_{z}=3 \mathrm{~nm}$, $n_{x}=15 \mathrm{~nm}$, and $n_{y}=6 \mathrm{~nm}$. Due to the magnetostatic interaction, the alternative pinned DWs adopt Bloch up $\left(\vec{m}_{\text {DW }} \propto+\vec{u}_{y}\right)$ and Bloch down $\left(\vec{m}_{\text {DW }} \propto-\vec{u}_{y}\right)$ magnetic configurations. A current source could be used to provide a fixed current along the strip. Under it, the central pinned DW rotates coherently counterclockwise. In the strip with three pinned DWs (Figure 11(b)), the ones at the left and at the right sides rotate clockwise. The same occurs for the strip containing five pinned DW (Figure 11(c)), where the ones at the left and the right extrema rotate similarly to the central one. Figure 11(e) depicts the temporal evolution of the induced voltage signal $V_{\text {smf }}(t)$ (in units of the polarization factor $P$ ) under a current density of $j_{a}=0.05 \mathrm{~A} / \mu \mathrm{m}^{2}$ for strips with several pinned DWs in the perfect adiabatic case. For this system, where all constrictions are equal, both $V_{\mathrm{dc}}$ and $V_{\mathrm{ac}}$ increase linearly with the number of pinned DWs (see Figure 11(f)), and, therefore, if the voltage signal 
can be experimentally detected, this proposed system could be useful to develop oscillators and/or amplifiers at the nanoscale.

However, there are a few aspects which have to taken into account and controlled before going to the real device. The first one is the separation $S$ between pinned DWs. When the constriction are placed close to each other, the Bloch configurations are not stable, and the DWs adopt Neel configurations magnetized along the strip axis. This is what happens for three DWs pinned at similar constrictions separated $S \leq 45 \mathrm{~nm}$, and due to the magnetostatic coupling, a small current of $j_{a}=0.05 \mathrm{~A} / \mu \mathrm{m}^{2}$ is not high enough to promote pinned DW oscillations. It was also verified that for separations $S \geq 130 \mathrm{~nm}$ the induced voltage signal does not change. Other important challenge is the limitation of the available nanolithography techniques. Nowadays, fabricating identical notches in a strip at the nanoscale is a very difficult art. Therefore, the influence of different constrictions, with different shape and sizes, on the induced voltage signal has to be analyzed. It was verified that the linear increasing of the oscillating contribution to the induced voltage signal $\left(V_{\mathrm{ac}}\right)$ is not preserved with the number of pinned DWs at irregular constrictions, but the direct contribution $\left(V_{\mathrm{dc}}\right)$ increases almost linearly with the number of pinned DWs even in the case of different constrictions [41]. Thermal effects are also relevant. For instance, the coherent oscillation of the internal DW magnetization can be only achieved at very low temperatures $(T \approx 10 \mathrm{~K})$ for the constrictions analysed here. It was also confirmed, by means of preliminary simulations, that the internal DW structure is also coherently oscillating under injected static currents at room temperature if the constriction is made deeper. The challenging point consists on filtering the noise due to thermal fluctuations from the induced voltage signal. All these numerical predictions claim for a future experimental verification. Conceptually, it is straightforward to detect the induced voltage due to these pinned DW oscillations by simply measuring the voltage difference between the ends of the strip. In practice, however, this is not a trivial task, and nowadays only Yang et al. [89] have reported experimental measures of this signal. Indeed, the contribution of the spin-motive force to the voltage signal due to the pinned DW oscillations is only one of several voltages arising from different effects. For instance, an experimental challenge is to sort out the $V_{\text {smf }}$ from other signals such as the voltage induced by the changing magnetic fluxes in the detection circuit as due to the classical Faraday's law. I hope that these theoretical predictions motivate experimental progress in this direction.

\section{Conclusions and Final Remarks}

The static and dynamics of DWs along thin rectangular cross-section strips with high PMA driven by spin-polarized currents has been theoretically studied from both micromagnetic $(\mu M)$ and one-dimensional (1 DM) models points of view. These materials are especially relevant for further technological applications because the intrinsic threshold current for current-induced DW motion can be drastically reduced with respect to soft materials such as Permalloy.
Thermal fluctuations and disorder (in particular, edge roughness) were both included in both models with the aim of providing a much more realistic description of experimental measurements. The current-induced DW propagation along a rough strip at room temperature was studied for both single layer strips and asymmetric multilayers stacks. In the former case, where the spin-orbit interaction (SOI) is negligible, the DW velocity increases firstly exponentially with current, following a creep regime which is governed by the pinning and thermal fluctuations. For very high currents, the DW mobility saturates reaching a value which approaches to the case of a perfect strip. It is interesting to note that, even when the nonadiabatic parameter is equal to the damping $(\xi=\alpha)$, the DW propagates by transforming its internal structure between Bloch and Neel configurations in both the creep and high-current regimes. When a ferromagnetic strip with high PMA is sandwiched between two asymmetric nonmagnetic in a multilayer stack, the SOI on the conductions electrons allows for the transfer of orbital angular momentum from the crystal lattice to the local magnetization, and it is mediated by a Rashba field. This field is equivalent to a homogeneous transverse field, and for positive and negative currents, promotes the stabilization of one of the Bloch configurations, either up or down, by raising the energy barrier against Neel transformation. Therefore, in asymmetric stacks, the linear high mobility regime is extended to higher currents, and consequently, it allows to achieve rigid DW propagation with higher velocities than the ones achieved in single layer strips. Moreover, the extended $1 \mathrm{DM}$ including the Rashba field along with thermal effects and disorder allows us to describe very recent experimental observations, not only qualitative but also quantitatively, with reduced computational effort. These architectures are promising for DW-based applications.

The pinning and depinning of a initially trapped DW in an artificial constrictions was also evaluated. Also in these processes the PMA strips present technological advantages over similar strips made of soft ferromagnetic materials. For the analysed geometry, the field required to promote the DW depinning in the absence of current is very high because the energy barrier required to overcome the pinning potential is around 19 times larger than the thermal energy at room temperature. Therefore, in the absence of driving force, the pinned DW remains stable against thermal fluctuations. The depinning field decreases linearly as the current is raised, and the slope of this declension slightly increases with the nonadiabaticity. On the other hand, under zero field in the perfect adiabatic limit, the critical depinning density current is around $0.4 \mathrm{~A} / \mu \mathrm{m}^{2}$ at zero temperature. This corresponds to a threshold current of $72 \mu \mathrm{A}$, which is small enough to minimize unwanted Joule heating effects. The study also indicates that the probability of the DW depinning under a given current changes abruptly from $0 \%$ to $100 \%$ in a narrow range of fields. This result points out that the DW depinning is highly selective, which is also relevant for further technological applications. These results indicate that engineering of pinning sites in thin strips of high PMA provides an efficient pathway to achieve both high stability against thermal fluctuations and low 
current-induced DW depinning and, therefore, it can find application on designing memory devices driven by static currents. Moreover, the theoretical formalism is also useful to elucidate the magnitude of the nonadiabatic parameter. In particular, our results are in good qualitative agreement with the experimental measurements by Ravelosona et al. [44] exploring the high pinning regime $B_{d} \gg \mu_{0} H_{K} / \alpha$ : the experimentally observed linear decreasing of the depinning current with increasing field is in good agreement with our simulations. In that experiment, the depinning current at zero field was found around $0.1 \mathrm{~A} / \mu \mathrm{m}^{2}$, which, in spite of the different materials and geometry, is in the same order of magnitude than our results. By simulating the same geometry and materials as these experiments, the stochastic $1 \mathrm{DM}$ can be directly adopted in order to gain a better description of the experimental observations by taking into account the effect of the constant temperature of the sample and, therefore, a more accurate estimate of both the polarization factor and the nonadiabatic parameter, along with their dependence on the temperature could be addressed. Much more experimental and numerical studies have to done to address this issue.

Finally, the possibility of engineering nano-oscillator based on pinned DWs was explored from a theoretical point of view. The pinned DW oscillations driven by direct current were described. Both frequency and averaged DW position increase with the applied current in the pinned oscillating regime. However, the DW position has also another alternating contribution with an amplitude which decreases with the applied current. The contribution due to the spinmotive force to the voltage signal induced by these pinned DW oscillations has been also characterized. It consists of both static and oscillating contributions. The first one increases linearly with the applied current and it does not depend on the nonadiabaticity. However, the oscillating contribution depends on the nonadiabatic parameter, and, therefore, information on the strength of the nonadiabaticity could be obtained by means of experimental measurements which could detect such an oscillating voltage signal. The induced voltage signal due to the DW oscillations in a strip with several pinned domain walls was also analysed. The theoretical prediction that spin-motive force voltage signal can be linearly enhanced with the number of walls could be useful to develop DW-based nano-oscillators. Some of the possible experimental challenges, such as the limitation in the nanolithography and detection of the voltage signal, were also discussed.

Before ending this review, it is worthy to enumerate the limitations of the adopted theoretical formalism and numerical models, along with the future tasks to be performed in the framework of the current-induced DW dynamics.

(i) The injected density current was assumed to be uniform along the strips. However, there must be an effect due to the spatial dependence of the injected current, specially in the analysis of the current-induced DW depinning from a constriction, where the current has to flow more densely than in the unconstricted zones. Taking into account the spatial dependence of the injected current requires to numerically solve the Laplace equation for the electrostatic potential with the corresponding boundary conditions. This can be done in a preliminary simulation, which them has to be included in the full micromagnetic modeling in order to elucidate its effect for each specific case.

(ii) The Oersted field due to the injected current has neither been taken into account. In the full micromagnetic model, this classical contribution can be straightforwardly introduced by numerically solving Biot-Savart's Law. When a uniform current density is injected along the axis $\left(\vec{j}_{a}=j_{a} \vec{u}_{x}\right)$ of an infinitely long and perfect strip with thin rectangular crosssection $\left(L_{z} \ll L_{y}\right)$, the Oersted field can be analytically evaluated. In this specific case, the Oersted field has a perpendicular $z$-component that is maximum at the strip edges and almost proportional to the strip thickness $\left(L_{z}\right)$. The $y$-component of this Oersted field is slightly smaller, and changes sign across the strip thickness so that for thin nanostrips this field should have virtually no effect. The $x$-component field component is zero, so that it has no direct effect on the DW position. The influence of the Oersted field for imperfect samples, for example, with an edge roughness or even a notch that reduces locally the nanostrip width, should be investigated in each particular case. For this purpose, the current density cannot be assumed as uniform, and it requires a separate evaluation as it was already pointed out.

(iii) It is also well known that the passage of an electric current through a conductor releases heat resulting in a rise of the temperature of the conductor. However, up to now this Joule heating as due to the injected current has not included in the simulations because it involves theoretical problems. From a numerical point of view, it is needed to solve the heat diffusion equation in order to deduce the local value of the temperature as function of the space and the elapsed time. This could be done straight forwardly, but after that we will face a theoretical problem. On the one hand, all micromagnetic parameters are temperature dependent $\left(M_{s}(T), A(T)\right.$, or $\left.P(T)\right)$. However, the micromagnetic approach assumes that temperature is far from the Curie temperature, and it considers constant material parameters. On the other hand, thermal effects are commonly included in the micromagnetic formalism by adding a random thermal field [75, 76], which is assumed to be a Gaussian distributed and both spacial and temporal uncorrelated white noise (see (2) and (3)). This Langevin formalism assumes that the temperature of the system is the same for all points in the sample. Therefore, if the temperature depends on the position along the strip, an alternative theoretical formalism should be developed.

All these three phenomena (nonuniform current, the Oersted field and the Joule heating) cannot be taken into 
account in the framework of the $1 \mathrm{DM}$, and, therefore, the study of their role on the current-induced DW dynamics will require sophisticated and time-consuming micromagnetic simulations. Developing efficient micromagnetic tools including these fundamental effects would provide important steps towards the fully understanding of the DW dynamics driven by spin-polarized currents.

\section{Acknowledgments}

This work was supported by Project MAT2011-28532-C0301 from Spanish government. The author thanks M. Miron and G. Gaudin for providing their experimental data of Figure 6.

\section{References}

[1] A. Hubert and R. Schafer, Magnetic Domains. The Analysis of Magnetic Microstructures, Springer, 1998.

[2] L. Berger, "Low-field magnetoresistance and domain drag in ferromagnets," Journal of Applied Physics, vol. 49, article 2156, 6 pages, 1978.

[3] L. Berger, "Exchange interaction between ferromagnetic domain wall and electric current in very thin metallic films," Journal of Applied Physics, vol. 55, article 1954, 3 pages, 1984.

[4] S. Zhang, P. M. Levy, and A. Fert, "Mechanisms of spinpolarized current-driven magnetization switching," Physical Review Letters, vol. 88, no. 23, Article ID 236601, 2004.

[5] S. Zhang and Z. Li, "Roles of nonequilibrium conduction electrons on the magnetization dynamics of ferromagnets," Physical Review Letters, vol. 93, no. 12, Article ID 127204, 2004.

[6] G. Tatara and H. Kohno, "Theory of current-driven domain wall motion: spin transfer versus momentum transfer," Physical Review Letters, vol. 92, no. 8, Article ID 086601, 2004.

[7] X. Waintal and M. Viret, "Current-induced distortion of a magnetic domain wall," Europhysics Letters, vol. 65, no. 3, pp. 427-433, 2004.

[8] A. Thiaville, Y. Nakatani, J. Miltat, and Y. Suzuki, "Micromagnetic understanding of current-driven domain wall motion in patterned nanowires," Europhysics Letters, vol. 69, no. 6, pp. 990-996, 2005.

[9] J. Grollier, D. Lacour, V. Cros et al., "Switching the magnetic configuration of a spin valve by current-induced domain wall motion," Journal of Applied Physics, vol. 92, no. 8, p. 4825, 2002.

[10] N. Vernier, D. A. Allwood, D. Atkinson, M. D. Cooke, and R. P. Cowburn, "Domain wall propagation in magnetic nanowires by spin-polarized current injection," Europhysics Letters, vol. 65, no. 4, pp. 526-532, 2004.

[11] M. Kläui, C. A. F. Vaz, J. A. C. Bland et al., "Controlled and reproducible domain wall displacement by current pulses injected into ferromagnetic ring structures," Physical Review Letters, vol. 94, no. 10, Article ID 106601, 2005.

[12] G. S. D. Beach, C. Knutson, C. Nistor, M. Tsoi, and J. L. Erskine, "Nonlinear domain-wall velocity enhancement by spin-polarized electric current," Physical Review Letters, vol. 97, no. 5, Article ID 057203, 2006.

[13] M. Laufenberg, W. Buhrer, D. Bedau et al., "Temperature dependence of the spin torque effect in current-induced domain wall motion," Physical Review Letters, vol. 97, no. 4, Article ID 046602, 4 pages, 2006.
[14] M. Hayashi, L. Thomas, C. Rettner, R. Moriya, X. Jiang, and S. S. P. Parkin, "Dependence of current and field driven depinning of domain walls on their structure and chirality in permalloy nanowires," Physical Review Letters, vol. 97, no. 20, Article ID 207205, 2006.

[15] L. Thomas, M. Hayashi, X. Jiang, R. Moriya, C. Rettner, and S. S. P. Parkin, "Oscillatory dependence of currentdriven magnetic domain wall motion on current pulse length," Nature, vol. 443, no. 7108, pp. 197-200, 2006.

[16] M. Hayashi, L. Thomas, C. Rettner, R. Moriya, Y. B. Bazaliy, and S. S. P. Parkin, "Current driven domain wall velocities exceeding the spin angular momentum transfer rate in permalloy nanowires," Physical Review Letters, vol. 98, no. 3, Article ID 037204, 2007.

[17] L. Thomas, M. Hayashi, X. Jiang, R. Moriya, C. Rettner, and S. Parkin, "Resonant amplification of magnetic domain-wall motion by a train of current pulses," Science, vol. 315, no. 5818, pp. 1553-1556, 2007.

[18] D. Bedau, M. Klaui, S. Krzyk, U. Rudiger, G. Faini, and L. Vila, "Detection of current-induced resonance of geometrically confined domain walls," Physical Review Letters, vol. 99, no. 14, Article ID 146601, 2007.

[19] G. Meier, M. Bolte, R. Eiselt, B. Kruger, P. Dong-Hyun, and K. Fischer, "Direct imaging of stochastic domain-wall motion driven by nanosecond current pulses," Physical Review Letters, vol. 98, no. 18, Article ID 187202, 2007.

[20] S. Laribi, V. Cros, M. Muoz et al., "Reversible and irreversible current induced domain wall motion in $\mathrm{CoFeB}$ based spin valves stripes," Physical Review Letters, vol. 90, no. 23, Article ID 232505, 3 pages, 2007.

[21] M. Y. Im, L. Bocklage, P. Fischer, and G. Meier, "Direct observation of stochastic domain-wall depinning in magnetic nanowires," Physical Review Letters, vol. 102, no. 14, Article ID 147204, 2009.

[22] S. Lepadatu, A. Vanhaverbeke, D. Atkinson, R. Allenspanch, and C. H. Marrow, "Dependence of domain-wall depinning threshold current on pinning profile," Physical Review Letters, vol. 102, no. 12, Article ID 127203, 4 pages, 2009.

[23] S. G. S. D. Beach, M. Tsoi, and J. L. Erskine, "Current-induced domain wall motion," Journal of Magnetism and Magnetic Materials, vol. 320, no. 7, pp. 1272-1281, 2008.

[24] R. A. Duine, A. S. Nunez, and A. H. MacDonald, "Thermally assisted current-driven domain-wall motion," Physical Review Letters, vol. 98, no. 5, Article ID 056605, 2007.

[25] A. Mougin, M. Cormier, J. P. Adam, P. J. Metaxas, and J. Ferre, "Domain wall mobility, stability and Walker breakdown in magnetic nanowires," Europhysics Letters, vol. 78, no. 5, Article ID 57007, 2007.

[26] E. Martinez, L. Lopez-Diaz, L. Torres, C. Tristan, and O. Alejos, "Thermal effects in domain wall motion: Micromagnetic simulations and analytical model," Physical Review B, vol. 75, no. 17, Article ID 174409, 11 pages, 2007.

[27] E. Martinez, L. Lopez-Diaz, O. Alejos, L. Torres, and C. Tristan, "Thermal effects on domain wall depinning from a single notch," Physical Review Letters, vol. 98, no. 26, Article ID 267202, 2007.

[28] E. Martinez, L. Lopez-Diaz, O. Alejos, and L. Torres, "Resonant domain wall depinning induced by oscillating spinpolarized currents in thin ferromagnetic strips," Physical Review B, vol. 77, no. 14, Article ID 144417, 2008.

[29] E. Martinez, L. Lopez-Diaz, O. Alejos, L. Torres, and M. Carpentieri, "Domain-wall dynamics driven by short pulses along thin ferromagnetic strips: micromagnetic simulations and 
analytical description," Physical Review B, vol. 79, no. 9, Article ID 094430, 14 pages, 2009.

[30] O. Boulle, G. Malinowski, and M. Klaui, "Current-induced domain wall motion in nanoscale ferromagnetic elements," Materials Science and Engineering R, vol. 72, no. 9, pp. 159187, 2011.

[31] D. A. Allwood, G. Xiong, M. D. Cooke et al., "Submicrometer ferromagnetic NOT gate and shift register," Science, vol. 296, no. 5575, pp. 2003-2006, 2002.

[32] D. A. Allwood, G. Xiong, C. C. Faulkner, D. Atkinson, D. Petit, and R. P. Cowburn, "Magnetic domain-wall logic," Science, vol. 309, no. 5741, pp. 1688-1692, 2005.

[33] P. Xu, K. Xia, C. Gu, L. Tang, H. Yang, and J. Li, "An all-metallic logic gate based on current-driven domain wall motion," Nature Nanotechnology, vol. 3, no. 2, pp. 97-100, 2008.

[34] S. S. P. Parkin, "Shiftable magnetic shift register and method of using the same," U.S. Patent No. 6834005, 2004.

[35] S. S. P. Parkin, M. Hayashi, and L. Thomas, "Magnetic domain-wall racetrack memory," Science, vol. 320, no. 5873, pp. 190-194, 2008.

[36] D. Atkinson, D. S. Eastwood, and L. K. Bogart, "Controlling domain wall pinning in planar nanowires by selecting domain wall type and its application in a memory concept," Applied Physics Letters, vol. 92, no. 2, Article ID 022510, 3 pages, 2008.

[37] J. He and S. Zhang, "Localized steady-state domain wall oscillators," Applied Physics Letters, vol. 90, no. 14, Article ID $142508,2007$.

[38] T. Ono and Y. Nakatani, "Magnetic domain wall oscillator," Applied Physics Express, vol. 1, Article ID 061301, 2008.

[39] L. Berger, "Possible existence of a Josephson effect in ferromagnets," Physical Review B, vol. 33, no. 3, pp. 1572-1578, 1986.

[40] A. Bisig, L. Heyne, O. Boulle, and M. Klaui, “Tunable steadystate domain wall oscillator with perpendicular magnetic anisotropy," Applied Physics Letters, vol. 95, Article ID 162504, 2009.

[41] E. Martinez, L. Torres, and L. Lopez-Diaz, "Oscillator based on pinned domain walls driven by direct current," Physical Review B, vol. 83, no. 17, Article ID 174444, 10 pages, 2011.

[42] E. Martinez, G. Finocchio, and M. Carpentieri, "Stochastic resonance of a domain wall in a stripe with two pinning sites," Applied Physics Letters, vol. 98, no. 7, Article ID 072507, 3 pages, 2011.

[43] D. Ravelosona, F. Caysol, J. Wunderlich et al., "Dynamics of magnetization reversal in a mesoscopic wire," Journal of Magnetism and Magnetic Materials, vol. 249, no. 1-2, pp. 170174, 2002.

[44] D. Ravelosona, D. Lacour, J. A. Katine, B. D. Terris, and C. Chappert, "Nanometer scale observation of high efficiency thermally assisted current-driven domain wall depinning," Physical Review Letters, vol. 95, no. 11, Article ID 117203, 4 pages, 2005.

[45] D. Ravelosona, S. Mangin, J. A. Katine, E. E. Fullerton, and B. D. Terris, "Threshold currents to move domain walls in films with perpendicular anisotropy," Applied Physics Letters, vol. 90, no. 7, Article ID 072508, 3 pages, 2007.

[46] C. Burrowes, D. Ravelosona, C. Chappert et al., "Role of pinning in current driven domain wall motion in wires with perpendicular anisotropy," Applied Physics Letters, vol. 93, no. 17, Article ID 172513, 2008.

[47] T. A. Moore, I. M. Miron, G. Gaudin et al., "High domain wall velocities induced by current in ultrathin $\mathrm{Pt} / \mathrm{Co} / \mathrm{AlOx}$ wires with perpendicular magnetic anisotropy," Applied Physics Letters, vol. 93, no. 26, Article ID 262504, 3 pages, 2008.
[48] O. Boulle, J. Kimling, P. Warnicke et al., "Nonadiabatic spin transfer torque in high anisotropy magnetic nanowires with narrow domain walls," Physical Review Letters, vol. 101, no. 21, Article ID 216601, 2008.

[49] T. Koyama, G. Yamada, H. Taniwaga et al., "Control of domain wall position by electrical current in structured $\mathrm{Co} / \mathrm{Ni}$ wire with perpendicular agnetic anisotropy," Applied Physics Express, vol. 1, Article ID 101303, 3 pages, 2008.

[50] H. Tanigawa, K. Kondou, T. Koyama et al., "Current-driven domain wall motion in CoCrPt wires with perpendicular magnetic anisotropy," Applied Physics Express, vol. 1, no. 1, Article ID 011301, 2008.

[51] I. M. Miron, P. J. Zermatten, G. Gaudin, S. Auffret, B. Rodmacq, and A. Schuhl, "Domain wall spin torquemeter," Physical Review Letters, vol. 102, no. 13, Article ID 137202, 2009.

[52] L. San Emeterio Alvarez, K. Y. Wang, S. Lepadatu, S. Landi, S. J. Bending, and C. H. Marrows, "Spin-transfer-torque-assisted domain-wall creep in a Co/Pt multilayer wire," Physical Review Letters, vol. 104, no. 13, Article ID 137205, 2010.

[53] C. Burrowes, A. P. Mihai, D. Ravelosona et al., "Nonadiabatic spin-torques in narrow magnetic domain walls," Nature Physics, vol. 6, no. 1, pp. 17-21, 2010.

[54] H. Tanigawa, T. Koyama, G. Yamada et al., "Domain wall motion induced by electric current in a perpendicularly magnetized Co/Ni nano-wire," Applied Physics Express, vol. 2, no. 5, Article ID 053002, 2009.

[55] I. M. Miron, T. Moore, H. Szambolics et al., "Fast currentinduced domain-wall motion controlled by the Rashba effect," Nature Materials, vol. 10, no. 6, pp. 419-423, 2011.

[56] M. Cormier, A. Mougin, J. Ferre et al., "Fast propagation of weakly pinned domain walls and current-assisted magnetization reversal in $\mathrm{He}^{+}$-irradiated $\mathrm{Pt} / \mathrm{Co} / \mathrm{Pt}$ nanotracks," Journal of Physics D, vol. 44, no. 21, Article ID 215002, 2011.

[57] S. Fukami, T. Suzuki, Y. Nakatani et al., "Current-induced domain wall motion in perpendicularly magnetized $\mathrm{CoFeB}$ nanowire," Applied Physics Letters, vol. 98, no. 8, Article ID 082504, 3 pages, 2011.

[58] S. Fukami, T. Suzuki, Y. Nakatani et al., "Current-induced domain wall motion in perpendicularly magnetized $\mathrm{CoFeB}$ nanowire," Applied Physics Letters, vol. 98, no. 8, Article ID 082504, 2011.

[59] K. Ueda, T. Koyama, D. Chiba et al., "Current-induced magnetic domain wall motion in $\mathrm{Co} / \mathrm{Ni}$ nanowire at low temperature," Applied Physics Express, vol. 4, no. 6, Article ID 063003, 2011.

[60] J. P. Adam, N. Vernier, J. Ferre et al., "Nonadiabatic spintransfer torque in (Ga,Mn)As with perpendicular anisotropy," Physical Review B, vol. 80, no. 19, Article ID 193204, 4 pages, 2009.

[61] A. Kanda, A. Suzuki, F. Masukura, and H. Ohno, "Domain wall creep in (Ga,Mn)As," Applied Physics Letters, vol. 97, no. 3, Article ID 032504, 3 pages, 2010.

[62] S.-W. Jung, W. Kim, T.-D. Lee, K.-J. Lee, and H.-W. Lee, "Current-induced domain wall motion in a nanowire with perpendicular magnetic anisotropy," Applied Physics Letters, vol. 92, no. 20, Article ID 202508, 3 pages, 2008.

[63] T. Suzuki, S. Fukami, N. Ohshima, K. Nagahara, and N. Ishiwata, "Analysis of current-driven domain wall motion from pinning sites in nanostrips with perpendicular magnetic anisotropy," Applied Physics Letters, vol. 103, no. 11, Article ID 113913, 6 pages, 2008.

[64] S. Fukami, T. Suzuki, N. Ohshima, K. Nagahara, and N. Ishiwata, "Micromagnetic analysis of current driven domain 
wall motion in nanostrips with perpendicular magnetic anisotropy," Applied Physics Letters, vol. 103, no. 7, Article ID 07E718, 2008.

[65] J.-V. Kim and C. Burrowes, "Influence of magnetic viscosity on domain wall dynamics under spin-polarized currents," Physical Review B, vol. 80, no. 21, Article ID 214424, 8 pages, 2009.

[66] E. Martinez, L. Lopez-Diaz, O. Alejos, and L. Torres, "Thermally activated domain wall depinning in thin strips with high perpendicular magnetocrystalline anisotropy," Journal of Applied Physics, vol. 106, no. 4, Article ID 043914, 5 pages, 2009.

[67] F. Garcia-Sanchez, H. Szambolics, A. P. Mihai et al., "Effect of crystalline defects on domain wall motion under field and current in nanowires with perpendicular magnetization," Physical Review B, vol. 81, no. 13, Article ID 134408, 7 pages, 2010.

[68] S. Emori and G. D. Beach, "Enhanced current-induced domain wall motion by tuning perpendicular magnetic anisotropy," Applied Physics Letters, vol. 98, no. 13, Article ID 132508, 3 pages, 2011.

[69] G. Consolo and E. Martinez, "The effect of dry friction on domain wall dynamics: a micromagnetic study," Journal of Applied Physics, vol. 111, no. 7, Article ID 07D312, 3 pages, 2012.

[70] E. Martinez, "The stochastic nature of the domain wall motion along high perpendicular anisotropy strips with surface roughness," Journal of Physics, vol. 24, Article ID 024206, 2012.

[71] E. Martinez, "The influence of the Rashba field on the currentinduced domain wall dynamics: a full micromagnetic analysis, including surface roughness and thermal effects," Journal of Applied Physics, vol. 111, no. 7, Article ID 07D302, 3 pages, 2012.

[72] E. Martinez, "Micromagnetic analysis of the Rashba field on current-induced domain wall propagation," Journal of Applied Physics, vol. 111, no. 3, Article ID 033901, 6 pages, 2012.

[73] D. Weller, A. Moser, L. Folks et al., "High ku materials approach to 100 gbits/in2," IEEE Transactions on Magnetics, vol. 36 , no. 1, pp. 10-15, 2000.

[74] P. J. Metaxas, J. P. Jamet, A. Mougin et al., "Creep and flow regimes of magnetic domain-wall motion in ultrathin $\mathrm{Pt} /$ Co/Pt films with perpendicular anisotropy," Physical Review Letters, vol. 99, no. 21, Article ID 217208, 2007.

[75] W. F. Brown, "Thermal fluctuations of a single-domain particle," Physical Review, vol. 130, no. 5, pp. 1677-1686, 1963.

[76] J. L. Garcia-Palacios and F. J. Lazaro, "Langevin-dynamics study of the dynamical properties of small magnetic particles," Physical Review B, vol. 58, no. 22, pp. 14937-14958, 1998.

[77] A. A. Thiele, "Steady-state motion of magnetic domains," Physical Review Letters, vol. 30, no. 6, pp. 230-233, 1973.

[78] T. W. Chiang, L. J. Chang, C. Yu et al., "Demonstration of edge roughness effect on the magnetization reversal of spin valve submicron wires," Applied Physics Letters, vol. 97, no. 2, Article ID 022109, 3 pages, 2010.

[79] I. M. Miron, G. Gaudin, S. Auffret et al., "Current-driven spin torque induced by the Rashba effect in a ferromagnetic metal layer," Nature Materials, vol. 9, no. 3, pp. 230-234, 2010.

[80] A. Manchon and S. Zhang, "Theory of nonequilibrium intrinsic spin torque in a single nanomagnet," Physical Review B, vol. 78, no. 21, Article ID 212405, 4 pages, 2008.

[81] A. Manchon and S. Zhang, "Theory of spin torque due to spinorbit coupling," Physical Review B, vol. 79, no. 9, Article ID 094422, 9 pages, 2009.

[82] R. A. Duine, "Spin pumping by a field-driven domain wall," Physical Review B, vol. 77, no. 1, Article ID 014409, 5 pages, 2008.
[83] A. Stern, "Berry's phase, motive forces, and mesoscopic conductivity," Physical Review Letters, vol. 68, no. 7, pp. 10221025, 1992.

[84] C.-M. Ryu, "Spin motive force and faraday law for electrons in mesoscopic rings," Physical Review Letters, vol. 76, no. 6, pp. 968-970, 1996.

[85] S. E. Barnes and S. Maekawa, "Generalization of faraday's law to include nonconservative spin forces," Physical Review Letters, vol. 98, no. 24, Article ID 246601, 4 pages, 2007.

[86] W. M. Saslow, "Spin pumping of current in non-uniform conducting magnets," Physical Review B, vol. 76, no. 18, Article ID 184434, 14 pages, 2007.

[87] Y. Tserkovnyak and M. Mecklenburg, "Electron transport driven by nonequilibrium magnetic textures," Physical Review B, vol. 77, no. 13, Article ID 134407, 4 pages, 2008.

[88] R. A. Duine, "Effects of nonadiabaticity on the voltage generated by a moving domain wall," Physical Review B, vol. 79, no. 1, Article ID 014407, 8 pages, 2009.

[89] S. A. Yang, G. S. D. Beach, C. Knutson et al., "Universal electromotive force induced by domain wall motion," Physical Review Letters, vol. 102, no. 6, Article ID 067201, 4 pages, 2009. 

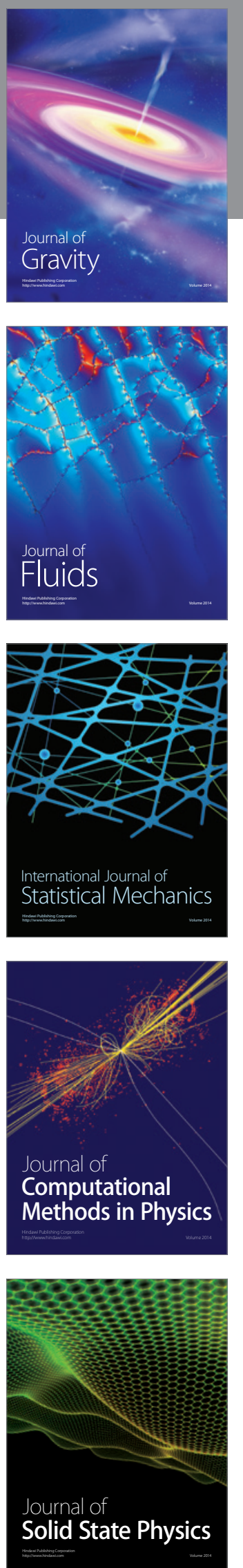

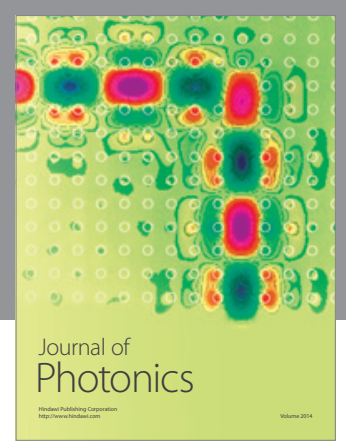

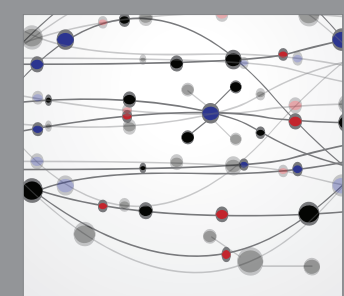

The Scientific World Journal
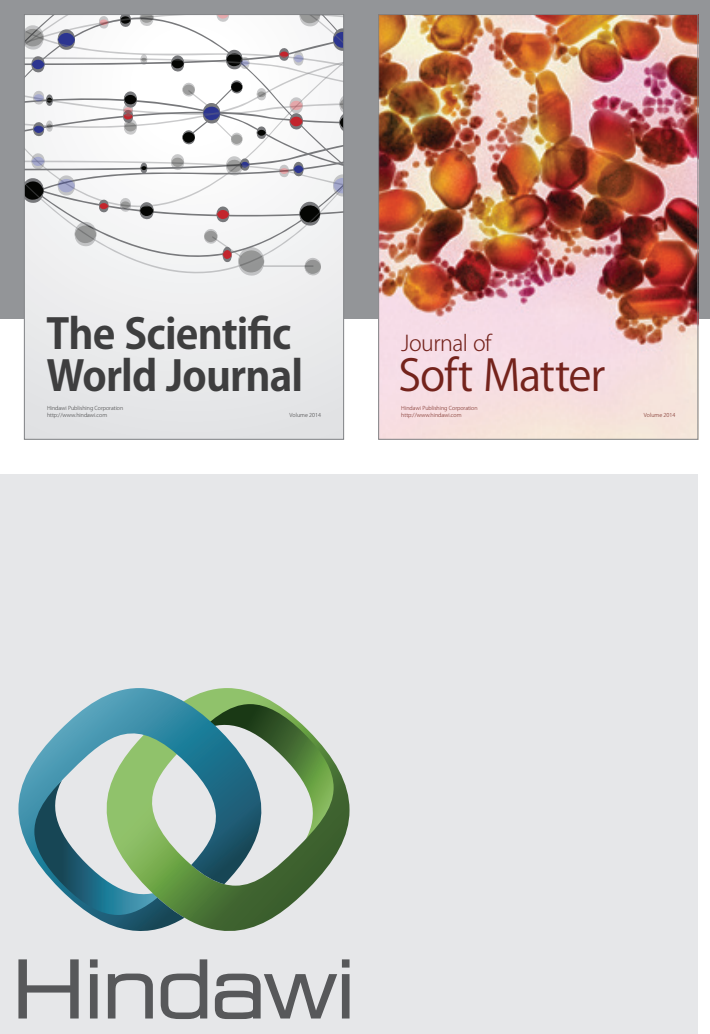

Submit your manuscripts at

http://www.hindawi.com
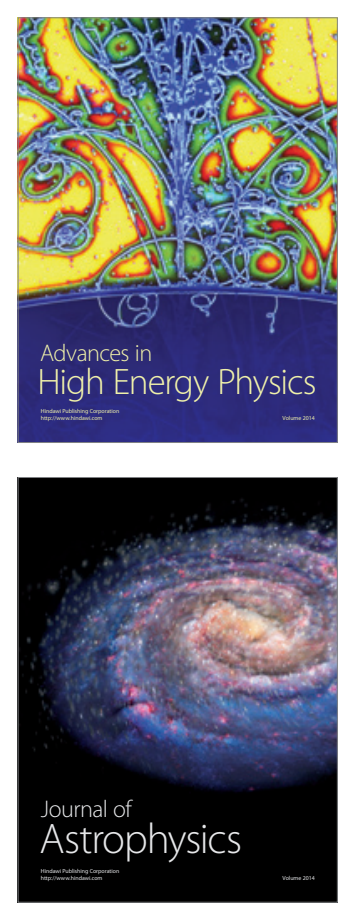
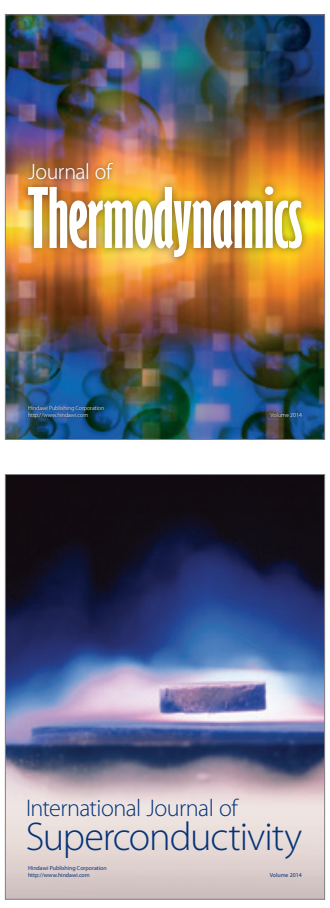
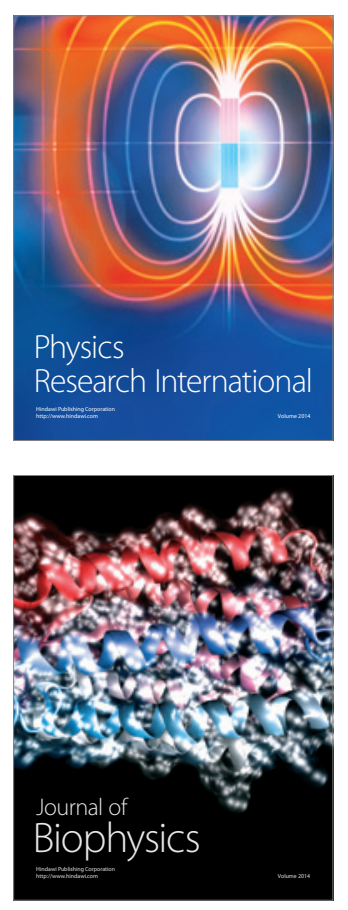
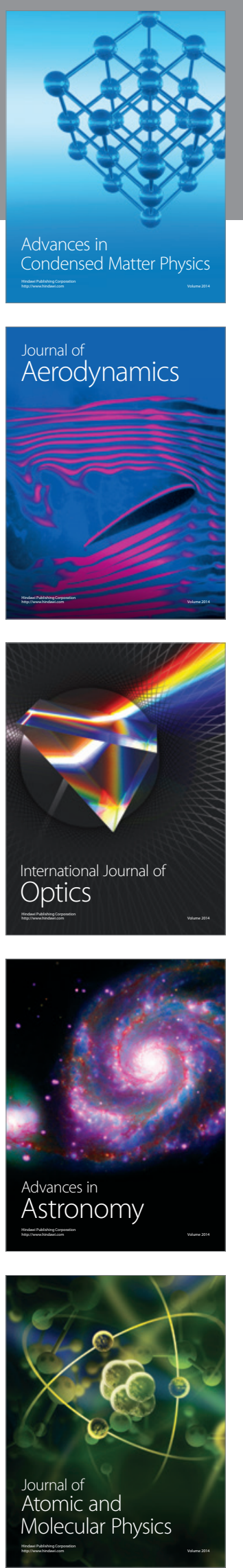